\title{
Improved Multiple Sequence Alignments using Coupled Pattern Mining
}

\author{
K. S. M. Tozammel Hossain, Debprakash Patnaik, Srivatsan Laxman, \\ Prateek Jain, Chris Bailey-Kellogg, and Naren Ramakrishnan
}

\begin{abstract}
We present ARMiCoRe, a novel approach to a classical bioinformatics problem, viz. multiple sequence alignment (MSA) of gene and protein sequences. Aligning multiple biological sequences is a key step in elucidating evolutionary relationships, annotating newly sequenced segments, and understanding the relationship between biological sequences and functions. Classical MSA algorithms are designed to primarily capture conservations in sequences whereas couplings, or correlated mutations, are well known as an additional important aspect of sequence evolution. (Two sequence positions are coupled when mutations in one are accompanied by compensatory mutations in another). As a result, better exposition of couplings is sometimes one of the reasons for hand-tweaking of MSAs by practitioners. ARMiCoRe introduces a distinctly pattern mining approach to improving MSAs: using frequent episode mining as a foundational basis, we define the notion of a coupled pattern and demonstrate how the discovery and tiling of coupled patterns using a max-flow approach can yield MSAs that are better than conservation-based alignments. Although we were motivated to improve MSAs for the sake of better exposing couplings, we demonstrate that our MSAs are also improvements in terms of traditional metrics of assessment. We demonstrate the effectiveness of ARMiCoRe on a large collection of datasets.
\end{abstract}

Index Terms-Multiple sequence alignment, coupled residues, pattern set mining, coupled patterns, max-flow problems.

\section{INTRODUCTION}

$\mathbf{E}$ VOLUTIONARY pressures on genes and proteins have constrained their (DNA and protein) sequences over generations. As organisms evolve through sequence modifications, mutations that have been evolutionarily selected for survival would be preserved in the sequence record. It is hence of intrinsic biological interest to inspect the sequence record and to unravel those mutations that have withstood the test of time and have been beneficial to the species.

Multiple sequence alignment (MSA) of biological sequences is a classical approach to understand evolutionary constraints. It has been said that "one or two homologous sequences whisper, ..., a full [MSA] shouts out loud" [1]. There is a plethora of MSA algorithms exists, with origins ranging

K. S. M. T. Hossain and N. Ramakrishnan are with the Department of Computer Science, Virginia Tech, Blacskburg, VA, 24060, USA.

E-mail: tozammel@vt.edu and naren@cs.vt.edu

D. Patnaik is with Amazon Inc., Seattle, WA 98109, USA.

E-mail: patnaik@vt.edu

S. Laxman and P. Jain are with Microsoft Research, Bangalore 560080, India.

E-mail: slaxman,prajain@microsoft.com

C. Bailey-Kellogg is with the Department of Computer Science, Dartmouth College, Hanover, NH 03755, USA.

E-mail: cbk@cs.dartmouth.edu from discrete algorithms [2] to probabilistic models, such as HMMs [3].

\section{A. Isn't MSA a Solved Problem?}

Although sequence alignment has become a widely deployed tool in bioinformatics, practically every MSA algorithm (e.g., ClustalW [2], Muscle [4], T-Coffee [5], and more) is designed to model and expose conservation, which although being a key evolutionary constraint, does not capture the richness of how sequences evolve and diverge. As seen in Fig. 1, two key forms of constraints are conservation and coupling. Column 4 of Fig. 1 (d) illustrates a conserved column, i.e., all residues are 'W.' Columns 2 and 8 of Fig. 1 (d) illustrate coupling, or compensatory mutations: whenever column 2 is ' $\mathrm{L}$,' column 8 is ' $\mathrm{T}$ '; similarly whenever column 2 is ' $\mathrm{M}$,' column 8 is ' $\mathrm{S}$.' In a typical alignment (e.g., Fig. 1 (b)), conservations are manifest and couplings are obscured; in fact, it is often accepted practice for biologists to 'hand tweak' such an alignment to incorporate structural information about sequences and thus obtain a better alignment.

Such tweaking is still somewhat of a black art and requires significant domain expertise. We were motivated to design an automated approach to better expose couplings in an MSA; but in doing so, our approach also improves MSAs according to traditional measures of assessment.

\section{B. Contributions}

- We present Alignment Refinement by Mining Coupled Residues (ARMiCoRe), a pattern mining approach to the problem of multiple sequence alignment. Using frequent episode mining as a foundational basis, we define the notion of a coupled pattern that elucidates covarying residues. Such coupled patterns are inferred using a levelwise approach and subsequently 'tiled' using a max-flow algorithm. The tiling is then used to direct the adjustment of a conservation based alignment to capture covarying residues.

- ARMiCoRe can be viewed as a novel application of pattern set discovery [6] where the goal is not just to mine interesting patterns (which is the purview of pattern discovery) but to select among them to optimize a set-based measure. ARMiCoRe can be used to tweak alignments from any existing algorithm, to better expose couplings or correlated mutations.

- As multiple sequence alignment is an established topic in bioinformatics, we subject ARMiCoRe to a thorough experimental evaluation involving 108 protein families. We 


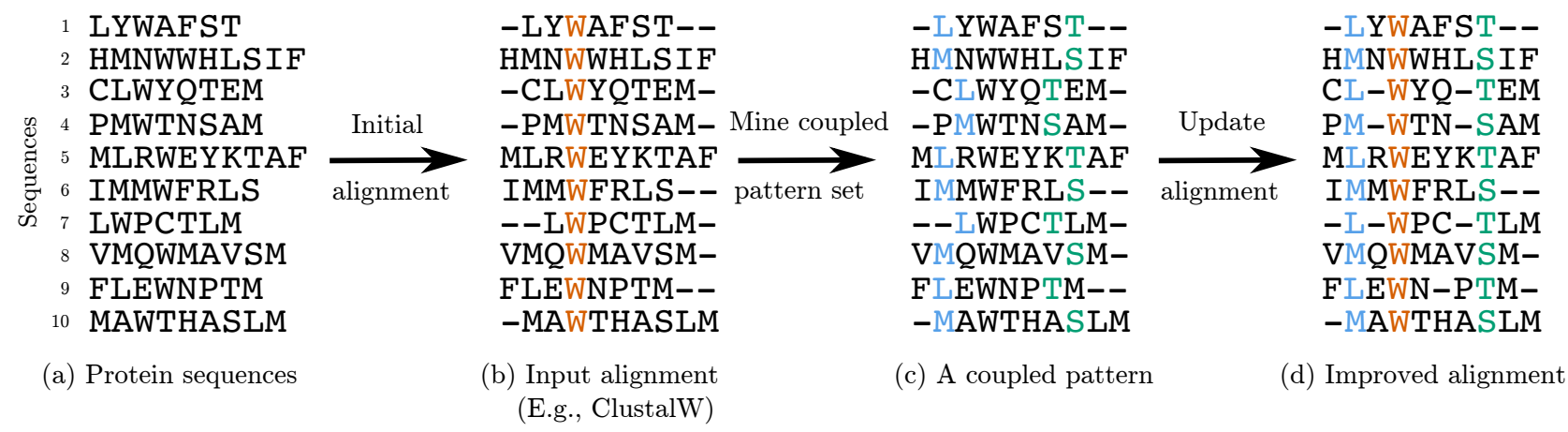

Fig. 1: Realignment of a hypothetical MSA using coupled pattern mining. Panel (b) is input to ARMiCoRe and (d) is the improved alignment.

identify selective superiorities of ARMiCoRe and demonstrate situations where it outperforms state-of-the-art MSA algorithms.

\section{RELATED WORK}

Multiple sequence alignment has been studied extensively for the past several decades (see [7], [8] for reviews). A rich set of features exist to classify MSA algorithms. These approaches fall broadly into two categories of alignment algorithms: global alignment vs. local alignment algorithms. Global alignment algorithms (e.g., ClustalW [2], MUSCLE [4], T-coffee [5], MAFFT [9], and ProbCons [10]) match sequences over their full lengths, whereas local alignment algorithms (e.g., DIALIGN [11], DIALIGN-T [12], and POA [13]) aim to align only the most similar regions between sequences. Local alignment is appropriate for sequence families where wellconserved regions are surrounded by variable regions. A second way to classify algorithms is in terms of the objective function (e.g., sum of pairs score, entropy, circular sum) used to identify the highest scoring alignment [8]. Finally, MSA algorithms can be classified based on their underlying optimization scheme: exact algorithms, progressive algorithms, and iterative algorithms. An exact algorithm attempts to simultaneously align all of the sequences and find an optimal alignment using an objective function [14]. The underlying problem has been proved to be NP-complete [15] and, hence, impractical for large numbers of sequences.

\section{A. Progressive and Iterative Algorithms:}

Heuristic approaches to MSA are either progressive or iterative algorithms. Progressive alignment algorithms (e.g., ClustalW [2] and T-Coffee [5] and LAGAN [16]), typically more appealing, involve building a guide tree based on sequence similarity and progressively aligning sequences following the order of the guide tree. Variants on progressive alignment typically use guide tree reestimation, modifying objective functions, and/or post-processing [8]. In guide tree reestimation, algorithms compute new distance matrices based on the initial MSA produced by progressive alignment, and the revised distance matrix is used to create a new guide tree. MAFFT [9], MUSCLE [4], PRIME [17], PRRP [18], MULTAN [19], and PROMALS [20] use this approach. Methods that modify the objective function are referred to as consistency-based methods, e.g., T-Coffee [5], DIALIGN [11], ProbCons [10], PCMA [21], and PROMALS [20]. The third variant involves post-processing, also known as iterative algorithms. In this approach, an alignment is first produced rapidly and then refined through a series of iterations until no more improvements can be made [8]. Examples are MUSCLE [4] and DIALIGN [11].

\section{B. Probabilistic Algorithms}

Probabilistic algorithms approach MSA by modeling different aspects: evolutionary models of indels , profile models, and hybrid models that combine probabilistic models with progressive alignment techniques. ProbCons [10] is a well known example that uses maximum expected accuracy scoring to infer a model and is especially useful for divergent sequences. A second example [3] uses a pair of HMMs as the scoring strategy.

\section{Constraint-based Algorithms}

These approaches (a.k.a. segment-based alignment algorithms) improve alignment quality by searching and incorporating information about homologs, conserved motifs/domains, and expert-supplied feedback about local similarity. Examples are COBALT [22], DIALIGN [11], DbClustal [23], and PROMALS [20].

As rich as the above landscape of MSA algorithms is, none of the above algorithms use covariation as a property to align sequences. Coupling is often viewed as a feature that 'comes out' of an alignment as opposed to a criterion or driver for computing the alignment. A very recent work, published in 2010 [24], is the lone exception which uses mutual information to detect coupled residues, and uses constraint programming to realign sequences. As we will show, ARMiCoRe captures not just coupled residues but the richer class of coupled patterns that tile the entire set of sequences; this greater expressiveness leads to improved MSAs, both in terms of exposing couplings, and in terms of traditional metrics of assessment (see Section V).

\section{FORMULATION}

We are given a collection $\mathcal{S}=\left\{s_{1}, \ldots, s_{n}\right\}$ of $n$ aligned sequences (or strings), each of length $m$, over a finite alphabet. 


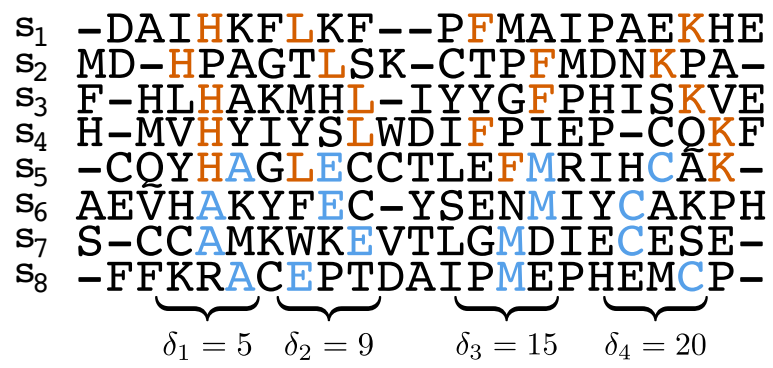

Fig. 2: Figure illustrating Example 2.

As shown in Fig. 1 (b), the sequences in $\mathcal{S}$ are assumed to have been aligned by a standard MSA method that typically favors conservation (and thus might contain gaps). Each sequence $s_{i}$, $i=1, \ldots, n$, can hence be expressed as $s_{i}=\left\langle E_{i 1}, \ldots, E_{i m}\right\rangle$, $E_{i j} \in \mathcal{E} \cup\{\varphi\}, j=1, \ldots, m$, where $\mathcal{E}$ denotes a finite alphabet and $\varphi$ is the gap symbol. In the case of DNA sequences, $\mathcal{E}=$ $\{A, C, T, G\}$, whereas for protein sequences, $\mathcal{E}$ comprises the 20 amino acid residues. We can even for instance denote amino acids by their physico-chemical properties so that the set of 20 amino acids can be reduced to a smaller set of properties.

Definition 1: An indexed pattern $\alpha$ (of size $\ell$ ) is defined by a pair of $\ell$-length sequences, $\left(\left\langle A_{1}^{\alpha}, \ldots, A_{\ell}^{\alpha}\right\rangle,\left\langle\delta_{1}^{\alpha}, \ldots, \delta_{\ell}^{\alpha}\right\rangle\right)$, where each $A_{j}^{\alpha} \in \mathcal{E}, \delta_{j}^{\alpha} \in \mathbb{Z}^{+}, j=1, \ldots, \ell$, and $\delta_{j+1}^{\alpha}>\delta_{j}^{\alpha}$, $j=1, \ldots,(\ell-1)$. We refer to $\left\langle\delta_{1}^{\alpha}, \ldots, \delta_{\ell}^{\alpha}\right\rangle$ as the sequence of positions over which $\alpha$ is defined.

The semantics of an indexed pattern $\alpha$ is essentially that in a sequence $s$ where $\alpha$ is said to occur, we expect that $A_{j}$ will appear at position $\delta_{j}$ (or very close to it) for every $1 \leq j \leq \ell$.

Definition 2: A sequence $s=\left\langle E_{1}, \ldots, E_{m}\right\rangle$ is said to contain an $\epsilon$-approximate occurrence of indexed pattern $\alpha$ if there exists a map $h:\{1, \ldots, \ell\} \rightarrow\{1, \ldots, m\}$, strictly increasing, such that $\forall j, 1 \leq j \leq \ell, E_{h(j)}=A_{j}^{\alpha}$ and $\left|h(j)-\delta_{j}^{\alpha}\right| \leq \epsilon$.

Example 1: $\alpha=(\langle A, E, M, C\rangle,\langle 5,9,15,20\rangle)$ is an indexed pattern of size $\ell=4$. An example sequence $s$ that contains an $\epsilon$-approximate occurrence of $\alpha$ is shown below (for $\epsilon=1$ ). Note that occurrences of symbols $A, E, M$ and $C$ can be found within 1 position of the locations 5, 9, 15 and 20 respectively.

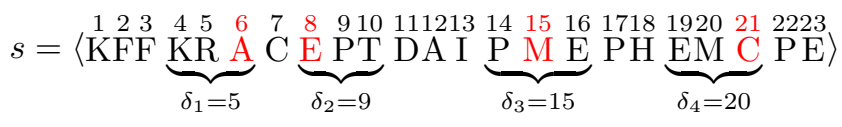

Definition 3: The $\epsilon$-support of an indexed pattern $\alpha$ over the collection $\mathcal{S}$ of sequences, denoted $f_{\epsilon}(\alpha)$, is the number of sequences in $\mathcal{S}$ that contain at least one $\epsilon$-approximate occurrence of $\alpha$; the corresponding set of $\epsilon$-supporting sequences is denoted by $\mathcal{U}_{\epsilon}(\alpha) \subseteq \mathcal{S}, f_{\epsilon}(\alpha)=\left|\mathcal{U}_{\epsilon}(\alpha)\right|$.

Definition 4: A coupled pattern, $\psi$, of size $k$ is defined as a $k$-tuple, $\left(\alpha_{1}, \ldots, \alpha_{k}\right)$, where each $\alpha_{i}, i=1, \ldots, k$ (referred to as a constituent of $\psi$ ) is an indexed pattern over a common sequence of positions $\left\langle\delta_{1}, \ldots, \delta_{\ell}\right\rangle$. The $\epsilon$-support of $\psi$ over a collection $\mathcal{S}$ of sequences, denoted $F_{\epsilon}(\psi)$, is defined as the total number of $\epsilon$-supporting sequences of its constituents found in $\mathcal{S}$, i.e., $F_{\epsilon}(\psi)=\left|\cup_{\alpha_{i} \in \psi} \mathcal{U}_{\epsilon}\left(\alpha_{i}\right)\right|$.
Example 2: Consider the collection of sequences, $\mathcal{S}=$ $\left\{s_{1}, \ldots, s_{8}\right\}$, defined in Figure 2. $\psi=\left(\alpha_{1}, \alpha_{2}\right)$ is an example coupled pattern of size 2, where $\alpha_{1}=(\langle H, L, F, K\rangle$, $\langle 5,9,15,20\rangle)$ and $\alpha_{2}=\langle A, E, M, C\rangle,\langle 5,9,15,20\rangle$ are indexed patterns over the same sequence of positions $\langle 5,9,15,20\rangle$. The $\epsilon$-support of $\psi$ over $\mathcal{S}$, for $\epsilon=1$, is $F_{1}(\psi)=8$.

Our main intuition here is that when there is enough evidence for a coupled pattern $\psi$ in a given data set $\mathcal{S}$, the associated sequence of positions $\left(\delta_{1}, \ldots, \delta_{\ell}\right)$ are coupled across multiple sequences of $\mathcal{S}$, in the sense that, mutations in one position are accompanied by corresponding mutations in the others. In Example 2, mutations of $H$ to $A$ in position 5, would be accompanied by three other mutations, namely, $L$ to $E$ in position $9, F$ to $M$ in position 15 and $K$ to $C$ in position 20. To facilitate the detection and measurement of the evidence for a coupled pattern, we define the notion of $\tau$-coverage with respect to the pattern's $\epsilon$-supporting sequences.

Definition 5: Let $\mathcal{S}$ be a given collection of sequences over $\mathcal{E} \cup\{\varphi\}$. Consider a coupled pattern $\psi=\left(\alpha_{1}, \ldots, \alpha_{k}\right)$ and its corresponding sets, $\mathcal{U}_{\epsilon}\left(\alpha_{i}\right), i=1, \ldots, k$, of $\epsilon$-supporting sequences. The $\tau$-coverage of $\psi$ in $\mathcal{S}$ with respect to its $\epsilon$ supporting sequences, denoted $\Gamma_{\epsilon}(\psi, \tau)$, is defined as follows:

$$
\Gamma_{\epsilon}(\psi, \tau)=\max _{\mathcal{D}_{1}, \ldots, \mathcal{D}_{k}} \sum_{i=1}^{k}\left|\mathcal{D}_{i}\right|
$$

where $\mathcal{D}_{i} \subset \mathcal{S}, i=1, \ldots, k$, such that the following hold: $\mathcal{D}_{i} \subset \mathcal{U}_{\epsilon}\left(\alpha_{i}\right), \mathcal{D}_{i} \cap \mathcal{D}_{j}$ is empty for $i \neq j$, and $\left|\mathcal{D}_{i}\right| \geq \tau$.

Essentially, we aim to compute mutually exclusive sets of $\epsilon$ supporting sequences for each of the $k$ constituents of $\psi$, such that each mutually exclusive set contains at least $\tau$ sequences, while the total number of distinct sequences in these sets is maximized. Mutual exclusiveness of sequences within a coupled pattern is necessary to prevent interactions between patterns in the realignment step. If a sequence containing two indexed patterns are given to the realigner, then there will be two constraints applied for the same position which may lead to unforeseeable results.

Example 3: For the same example as before, with $\epsilon=1$, we get the following sets of $\epsilon$-supporting sequences for $\alpha_{1}$ and $\alpha_{2}: \mathcal{U}_{\epsilon}\left(\alpha_{1}\right)=\left\{s_{1}, s_{2}, s_{3}, s_{4}, s_{5}\right\}\left(f_{1}\left(\alpha_{1}\right)=5\right)$ and $\mathcal{U}_{\epsilon}\left(\alpha_{2}\right)=$ $\left\{s_{5}, s_{6}, s_{7}, s_{8}\right\}\left(f 1\left(\alpha_{2}\right)=4\right)$. Setting $\mathcal{D}_{1}=\left\{s_{1}, s_{2}, s_{3}, s_{4}\right\}$ and $\mathcal{D}_{2}=\left\{s_{5}, s_{6}, s_{7}, s_{8}\right\}$ we get the 4 -coverage of $\psi$ with respect to its 1-supporting sequences to be $\Gamma_{1}(\psi, 4)=8$.

There are two main challenges in the detection and use of coupled patterns for improving multiple sequence alignment. First, given a data set $\mathcal{S}$ of (approximately aligned) sequences, we need to find coupled patterns which have high $\tau$-coverage over $\mathcal{S}$. Second, we need to use the high-coverage coupled patterns discovered to improve the MSA relative to the original alignment in $\mathcal{S}$.

Problem 1 (Mining Coupled Patterns): Consider a data set $\mathcal{S}$ of $m$-length sequences over $\mathcal{E} \cup\{\varphi\}$ and a fixed sequence of position indices, $\left\langle\delta_{1}, \ldots, \delta_{\ell}\right\rangle$. Given user-defined parameters, $\epsilon, K$ and $\tau$ (all non-negative integers) find a coupled pattern of size $k \leq K$ over $\left\langle\delta_{1}, \ldots, \delta_{\ell}\right\rangle$ which maximizes $\tau$-coverage with respect to its $\epsilon$-supporting sequences in $\mathcal{S}$. 


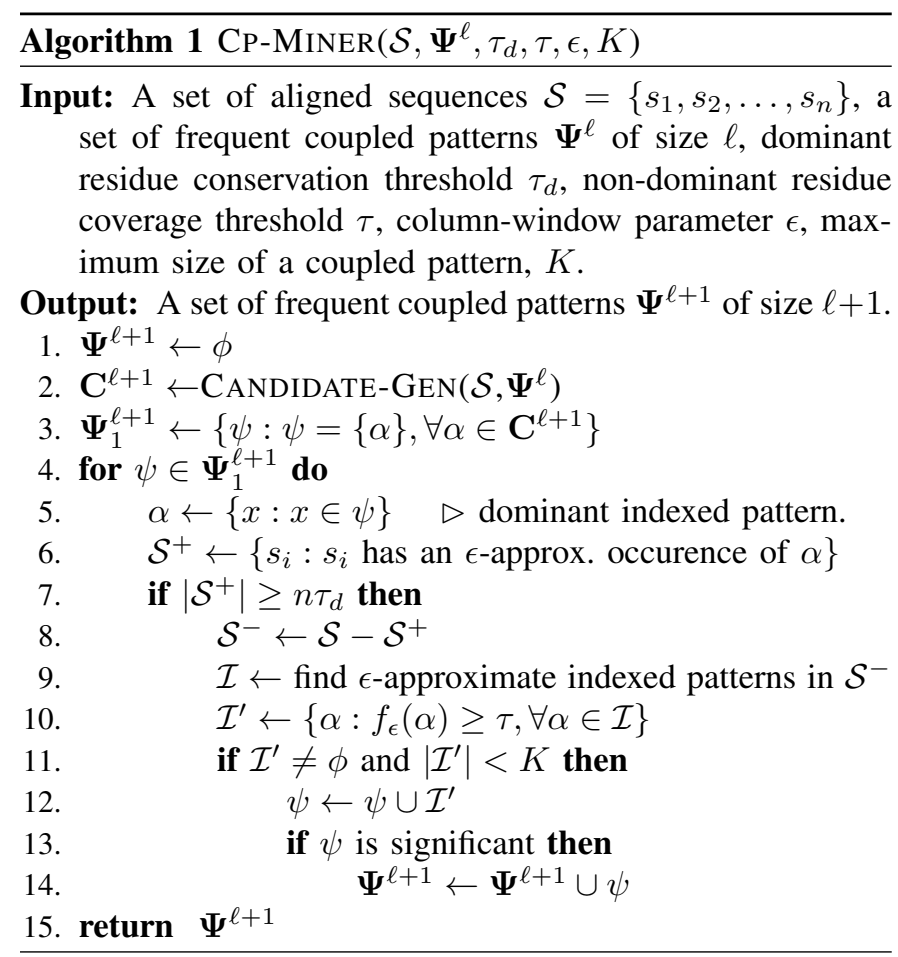

The MSA realignment problem can then be stated as follows.

Problem 2 (MSA Realignment): Given a data set $\mathcal{S}$ of $m$ length sequences over $\mathcal{E} \cup\{\varphi\}$ and a set of coupled patterns $\boldsymbol{\Psi}=\{\psi\}$ in $\mathcal{S}$ each of which has $\tau$-coverage of $\Gamma_{\epsilon}(\psi, \tau)=$ $\gamma$ over $\epsilon$-supporting sequences, find a realignment $\mathcal{S}^{\prime}$ of the sequences in $\mathcal{S}$ where all patterns in $\Psi$ have a $\tau$-coverage of $\Gamma_{\epsilon^{\prime}}(\psi, \tau) \geq \gamma$ for $\epsilon^{\prime}<\epsilon$.

In the above formulation, note that we require coupled patterns discovered in the original (approximate) alignment to still be manifest in the new alignment, but in a more obvious manner. Ideally $\epsilon^{\prime}=0$ (which is the situation for the example pattern in Fig. 1 (d)) but in practice we aim to obtain $\epsilon^{\prime}<\epsilon$.

\section{Algorithms}

In this section, we present ARMiCoRe, a new method for aligning multiple sequences based on coupling relationships that may exist between residues found in two or more sequence positions. The method consists of two main steps. We start by discovering high-support coupled patterns over various choices of position sequences (described in Sec. IV-A). Finally, in Sec. IV-C, we derive an alternative alignment $\mathcal{S}^{\prime}$ for $\mathcal{S}$ based on both the original ungapped sequences and the justdiscovered coupled patterns.

\section{A. Discovering Coupled Patterns}

The first step of ARMiCoRe is to choose the sequence positions over which to mine coupled patterns. Then standard level-wise methods (Apriori [26]) are used to discover coupled patterns (restricted to the chosen sequence positions) with sufficient support (cf. Sec. IV-A1). While level-wise searching for coupled patterns ARMiCoRe looks for patterns that have

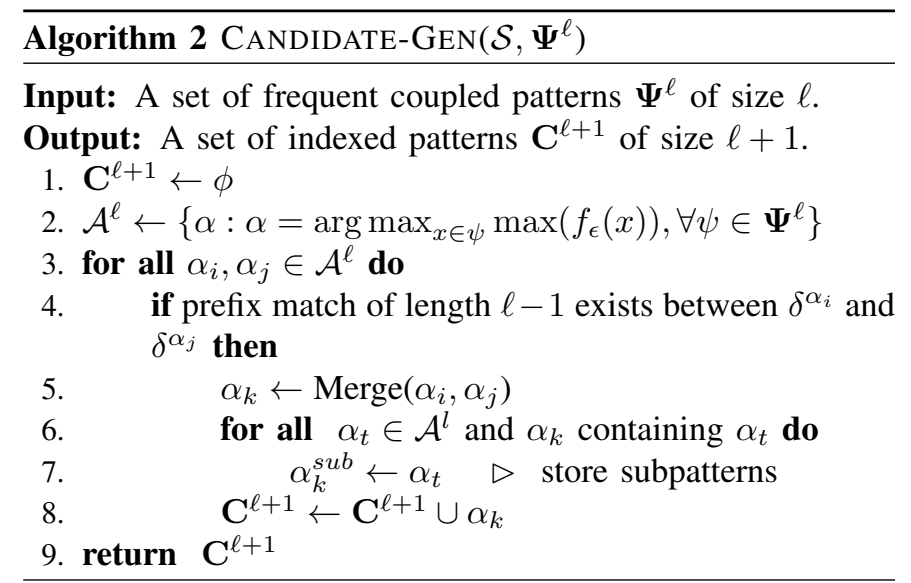

at most $K$ constituents ignoring $\tau$-coverage (cf. Sec. IV-A2). Then ARMiCoRe applies a statistical significance test to filter out uninteresting coupled patterns (cf. Sec. IV-A3). This gives us the pattern set, $\boldsymbol{\Psi}^{l}=\left\{\psi_{1}, \ldots, \psi_{|\Psi|}\right\}$, of $\ell$-size indexed patterns, each with support at least $\tau$, each has at most $K$ constituents, and each defined over a common sequence of positions, $\left\langle\delta_{1}, \ldots, \delta_{\ell}\right\rangle$. Each subset of indexed patterns in $\psi$ can thus be a potential candidate for a $\tau$-coverage coupled pattern. Finally, ARMiCoRe applies a max-flow approach to get the $\tau$-coverage of each $\psi$ (cf. Sec. IV-A4).

A lower-bound $\tau$ on the sizes $\left|\mathcal{D}_{i}\right|$ of the blocks corresponding to each constituent of a coupled pattern (see Definition 5) automatically enforces an upper-bound $\left\lfloor\frac{n}{\tau}\right\rfloor$ on the size, $k$, the coupled pattern. At first, it might appear as if the user only needs to prescribe $\tau$ to detect interesting patterns (since an upper-bound on $k$ is implied). However, we have observed that in the couplings that are already known in biological data sets, the number of constituents are typically far fewer than $\left\lfloor\frac{n}{\tau}\right\rfloor$. The notion of a coupling between two residue positions in biological datasets is based on the occurrences of a few amino acid combinations at these residue positions. If many amino acid combinations occur at two residue positions, the coupling between the two residue positions becomes weak. That is why protein families exhibiting couplings show a few amino acid combinations between two residue positions. If we set $\tau$ very low, then $K$ increases a lot, and it reduces the fidelity of the couplings at these positions. Hence, in our framework, the user must specify both an upper-bound $K$ for $k$ as well as a lower-bound $\tau$ on the block-sizes $\left|\mathcal{D}_{i}\right|$ of coupled patterns.

We now describe the steps in ARMiCoRe for finding a subset of indexed patterns that implies a coupled pattern, of size at most $K$, and which maximizes the $\tau$-coverage over its $\epsilon$-supporting sequences. The main hardness in the problem arises from having to maximize coverage with a $\tau$ constraint while restricting the number of constituent patterns to no more than $K$. Hence, we decouple the two problems and show that the individual problems can be solved efficiently. Specifically, we show that by ignoring the $\tau$ constraint, the problem of maximizing coverage is a sub-modular function-maximization problem with cardinality constraint. We propose Algorithm 1,2 for generating all possible coupled patterns of size at most $K$. 


\begin{tabular}{|c|c|}
\hline Cluster Id & Amino Acids \\
\hline 1 & APST \\
\hline 2 & ILVM \\
\hline 3 & C \\
\hline 4 & G \\
\hline 5 & ND \\
\hline 6 & RHKEQ \\
\hline 7 & FWY \\
\hline
\end{tabular}

(a)

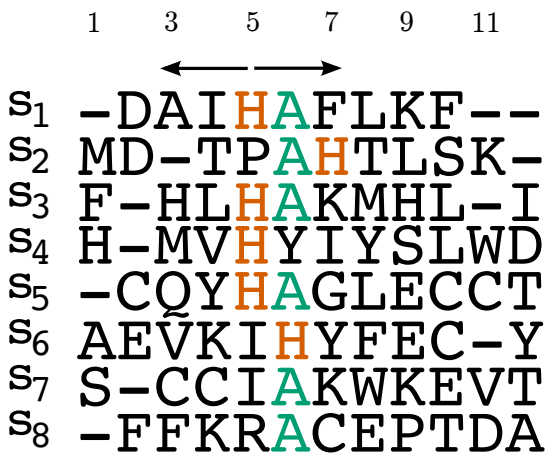

(b)

Fig. 3: (a) Clustering of amino acids proposed in [25]. (b) This figure describes window constraints. While looking for similar residue within a window the algorithm does not go beyond a conserved residue in a (semi)conserved column so that the (semi)conserved column is not distorted in the realignment process.

On the other hand, after selecting coupled patterns of size at most $K$, maximizing coverage with the $\tau$ constraint reduces to a max-flow problem.

1) Level-wise Coupled Pattern Mining: Our basic idea here is to organize the search for coupled patterns around the (semi) conserved columns of the current alignment. Level 1 patterns are comprised of individual columns, level 2 patterns are comprised of pairs of level 1 patterns, and so on.

For choosing a (semi) conserved column, we employ a dominant residue conservation threshold $\tau_{d}$ (see Line 7 of Algorithm 1). We use class-based conservation so that amino acid residues that have similar physico-chemical properties are considered conserved. Class-based conservation can be estimated using the Taylor diagram [27] or by k-means clustering of substitution matrices such as Blosum62 [25]. We have explored both approaches and found the latter to work better (with a setting of 7 non-overlapping clusters)(see Fig. 3a).

Amino acids in and around the semi-conserved columns (to within a window length of $\epsilon$ ) are organized into positive and negative sets of sequences describing the dominant combination and other, non-dominant, ones (see Fig. 4 (left)). While increasing the size of both the dominant and nondominant patterns for a column by searching for similar residues within a window for that column, the algorithm restricts itself to not go beyond a (semi)conserved column if it encounters any such column within the window. For example, in Fig. $3 b$ the column 5 is semiconserved and the residue ' $\mathrm{H}$ ' is the dominant residue in this column as it is the most frequent residue. The residue ' $\mathrm{H}$ ' at position 7 of sequence 2 is a candidate for extending the dominant pattern at column position 5 . As the column position 6 is almost fully conserved for residue 'A', the inclusion of ' $\mathrm{H}$ ' at position 7 of sequence 2 for the dominant pattern at column position 5 may destroy the conservation of column 6 in the realignment process. So the algorithm does not include ' $\mathrm{H}$ ' at position 7 of sequence 2 as a dominant residue for column 5. On the other hand, the algorithm will include the residue ' $\mathrm{H}$ ' at position 6 of the sequence 6 as a dominant residue for column 5 since this inclusion does not destroy the conservation of column 6 . As we construct level-2 and greater patterns, we take care to ensure that $\epsilon$ does not yield window lengths that cross another semi-conserved column.

2) High e-support using at most $K$ Constituents: We now present the approach taken by ARMiCoRe to solve the problem of maximizing coverage by enforcing only the upperbound $K$ (user-defined) on the number of constituents of $\psi$ while ignoring the $\tau$ constraint. We will test for $\tau$-coverage later as a post-processing step (see Sec. IV-A4). Note that at $\tau=0, \tau$-coverage is same as $\epsilon$-support, and this can be shown to be both monotonic and sub-modular with respect to its constituents. That is, if $\mathcal{A}$ and $\mathcal{B}$ are two subsets of $\psi$, such that $\mathcal{A} \subset \mathcal{B}$, then it can be shown that: $\Gamma_{\epsilon}(\mathcal{A} \cup \alpha, 0) \geq \Gamma_{\epsilon}(\mathcal{A}, 0)$, and, $\Gamma_{\epsilon}(\mathcal{A} \cup \alpha, 0)-\Gamma_{\epsilon}(\mathcal{A}, 0) \geq \Gamma_{\epsilon}(\mathcal{B} \cup \alpha, 0)-\Gamma_{\epsilon}(\mathcal{B}, 0)$. Consequently, we can use a greedy algorithm which guarantees a $\left(1-\frac{1}{e}\right)$-approximate solution [28]. In other words, we would find a subset of $\psi$ whose $\epsilon$-support (or 0-coverage) is within a factor of $\left(1-\frac{1}{e}\right)$ of the optimal subset.

3) Significance Testing of Coupled Patterns: For level-2 patterns and greater, we perform a 2-fold significance test, the first focusing on the dominant pattern and the second focusing on the non-dominant patterns. For a dominant pattern of size $l$, we compute the $p$-value for each dominant subpattern of size $l-1$ within the dominant pattern and then take the maximum as a $p$-value. For computing $p$-value we choose Poisson distribution if the number of sequences in the alignment is less than 100; otherwise, we choose binomial distribution. Let $p_{1}$ be the probability for the dominant residue in the subpattern of size $l-1$ and $p_{2}$ be the probability for the dominant residue in the column that is present in the original pattern but not in the subpattern. We use $n p$ as a parameter for the Poisson distribution and $n, p$ as parameters for the binomial distribution where $n$ is the number number of sequences in the alignment and $p=p_{1} * p_{2}$. For the non-dominant patterns, we conduct a standard enrichment analysis using the hypergeometric distribution to determine if the symbols in the non-dominant pattern are over-represented. The parameters for the hypergeometric distribution are the number of sequences in the alignment, the size of the non-dominant pattern, and the size of the union of all non-dominant patterns.

4) Checking $\tau$-coverage using Max-Flow: Once we have generated $\psi$ with high $\epsilon$-support we proceed to check if a 


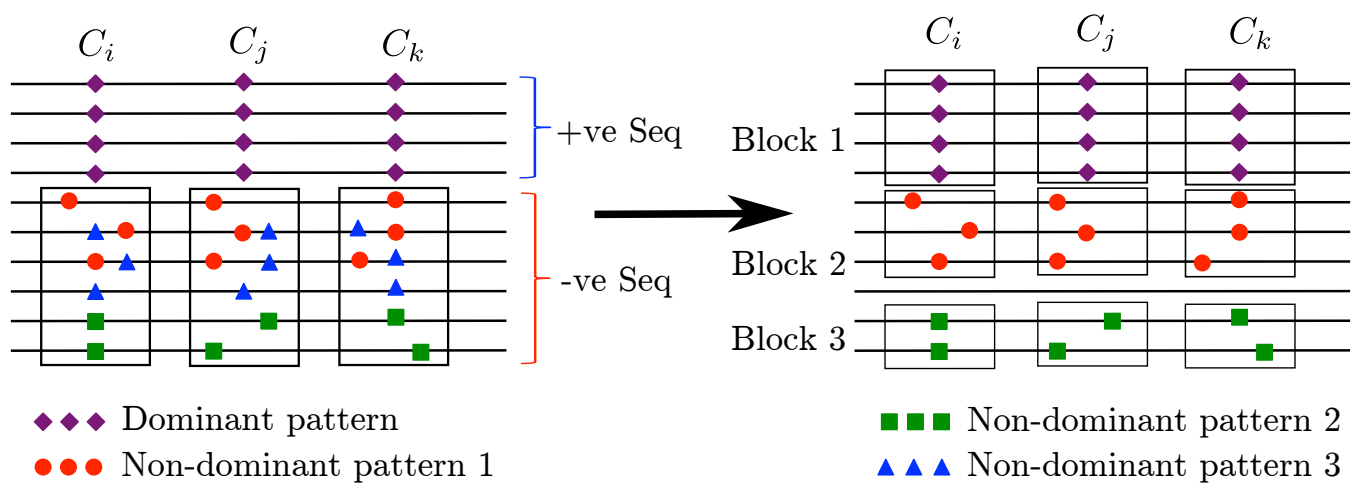

Fig. 4: Generating a coupled pattern set from all possible patterns. In the left figure a coupled pattern can be created from a dominant dominant pattern and three candidate non-dominant patterns that may overlap with each other. In right figure a possible construction of a coupled pattern consisting of one dominant pattern and two non-dominant pattern is shown.

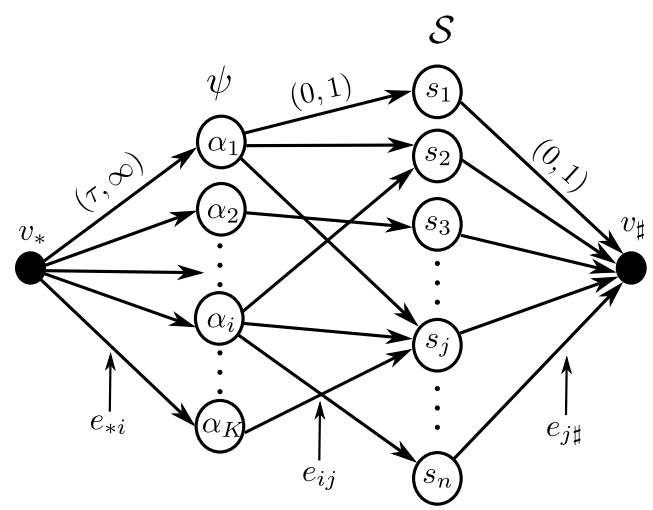

Fig. 5: Network $\mathcal{G}$ used in the max-flow step. Each $\alpha_{i}$ is an indexed pattern and each $s_{j}$ is a sequence. The nodes $v_{*}$ and $v_{\sharp}$ denote the source and the sink respectively. Each edge from $\alpha_{i}$ to $s_{j}$ has a flow of 1 if $s_{j}$ contains $\alpha_{i}$. The minimum flow from $v_{*}$ to an $\alpha_{i}$ is $\tau$ since $\alpha_{i}$ has a support of at least $\tau$.

non-zero $\tau$-coverage is feasible (Recall that the coverage will either be zero or the full $\epsilon$-support corresponding to the chosen subset of $\psi$ ). This problem reduces to a standard max-flow problem for which efficient (poly-time) algorithms exist. We now present the reduction of this problem to max-flow (see Fig. 5).

Let $\mathcal{G}=(\mathbf{V}, \mathbf{E})$ be a network with $v_{*}, v_{\sharp} \in \mathbf{V}$ denoting the source and sink of $\mathcal{G}$ respectively. In addition to $v_{*}$ and $v_{\sharp}$, there is a unique node in $\mathbf{V}$ corresponding to each indexed pattern $\alpha_{i} \in \psi$ and also to each sequence $s_{j} \in \mathcal{S}$, i.e., $\mathbf{V}=$ $\left\{v_{*}, v_{\sharp}\right\} \cup \psi \cup \mathcal{S}$. Three kinds of edges are in set $\mathbf{E}$ :

1) $e_{* i} \in \mathbf{E}$, representing an edge from the source node $v_{*}$ to the pattern node, $\alpha_{i} \in \mathbf{V}$. We will have $e_{* i} \in \mathbf{E}$, $\forall \alpha_{i} \in \psi$

2) $e_{j \sharp} \in \mathbf{E}$, representing an edge from the sequence node $s_{j} \in \mathbf{V}$ to the sink node $v_{\sharp}$. We will have $e_{j \sharp} \in \mathbf{E}$, $\forall s_{j} \in \mathcal{S}$

3) $e_{i j} \in \mathbf{E}$, representing an edge from pattern node $\alpha_{i} \in \mathbf{V}$ to the sequence node $s_{j} \in \mathcal{S}$, whenever the algorithm assigns $s_{j}$ to $\mathcal{D}_{i}$ (see Definition 5). We will have $e_{i j} \in$ $\mathbf{E}, \forall \alpha_{i} \in \psi, s_{j} \in \mathcal{S}$ such that $s_{j}$ is assigned to the block
$\mathcal{D}_{i}$ that corresponds to the $i^{\text {th }}$ pattern $\alpha_{i} \in \psi$.

For any edge $e \in \mathbf{E}$, let $\mathrm{LB}(e)$ and $\mathrm{UB}(e)$ denote, respectively, the lower and upper bounds on the capacity of edge $e$. Given a coupled pattern $\psi$, the computation of its $\tau$-coverage, $\Gamma_{\epsilon}(\psi, \tau)$, reduces to the computation of max-flow for the network $\mathcal{G}$ under the following capacity constraints:

1) $\mathrm{LB}\left(e_{* i}\right)=\tau, \mathrm{UB}\left(e_{* i}\right)=\infty, \forall \alpha_{i} \in \psi$

2) $\mathrm{LB}\left(e_{j \sharp}\right)=0, \mathrm{UB}\left(e_{j \sharp}\right)=1, \forall s_{j} \in \mathcal{S}$

3) $\mathrm{LB}\left(e_{i j}\right)=0, \mathrm{UB}\left(e_{i j}\right)=1, \forall \alpha_{i} \in \psi, s_{j} \in \mathcal{S}$

We can now use any max-flow algorithm, such as [29], [30] to obtain the max-flow in $\mathcal{G}$ subject to the stated capacity constraints. The flow returned will give us $\Gamma_{\epsilon}(\psi, \tau)$.

\section{B. Complexity Analysis}

The runtime for finding all possible coupled patterns depends on the number of sequences $(n)$, the alignment length $(m)$, the column-window threshold $(\epsilon)$, and the maximum size of the indexed pattern $(\ell)$. Let $p$ be the number of semiconserved columns found in level 1 indexed pattern mining. Then the running time for generating all possible coupled patterns is $\mathcal{O}\left(n m+l\left(p^{3}+l p^{2} n \epsilon\right)\right)$. Since $p \sim \mathcal{O}(m)$, the running time is $\mathcal{O}\left(l\left(m^{3}+l m^{2} n \epsilon\right)\right)$. Finding a $\tau$-coverage coupled pattern depends on the number of nodes $(\mathcal{O}(n+K))$ and the number of edges $(q)$ in the max-flow network for which the running time is $\mathcal{O}\left((n+K) q \log \left((n+K)^{2} / q\right)\right)$ [30].

\section{Updating the Alignment}

There are various ways to adjust the given alignment. One strategy that suggests itself is to modify the substitution matrix but this is not a good idea since this is a global approach and does not lend itself to the local shifting of columns as suggested by coupled pattern sets. We instead adopt a constraint-based alignment strategy, based on COBALT [22], which can flexibly incorporate domain knowledge. Constraints in COBALT are specified in terms of two segments from a pair of sequences that should be aligned with each other in the final result. To convert coupled patterns into constraints, we can adopt various strategies. One approach is to, for each pair of sequences, identify a pair of column positions that should be 
realigned based on the coupled pattern set. We then map these two positions in the alignment to the corresponding positions in the original (ungapped) sequences. (These two positions in terms of the original sequences thus constitute a segment pair of size one that should be realigned.) Taking all pairs of sequences in this manner would generate a huge number of constraints. We can reduce the number of constraints by considering consecutive pair of sequences. Another approach is to take a subset of sequences, say $S_{1}$, for whom the residues match over a column in the coupled pattern. We then take each of the sequences for whom residues do not match over that column in the coupled pattern, and create constraints by pairing the sequence with each of the sequences from $S_{1}$. COBALT guarantees a maximal consistent subset of these constraints to be occurred in the final alignment. The runtime for an alignment using COBALT is data-centric [22]. DIALIGN [31] is another possible algorithm that can be used to realign sequences. It takes user-defined anchor points but might yield non-aligned residues in the alignment. Due to our desire for global alignments we focus on he COBALT strategy but ARMiCoRe can be easily incorporated into DIALIGN as well.

\section{EXPERIMENTAL RESUlTS}

In this section, we assess ARMiCoRe on benchmark datasets. Due to space limitations, we provide only representative results illustrating selective superiorities of ARMiCoRe. Our goals are to answer the following questions:

1) How is the discovery of coupled patterns influenced by the dominant residue conservation threshold $\left(\tau_{d}\right)$, block coverage threshold $\tau$, and column window parameter $\epsilon$ ? (see Section V-C)

2) How does ARMiCoRe fare against classical algorithms on benchmark datasets? Here we choose ClustalW and COBALT, two representative MSA algorithms. (see Section V-D)

3) Can ARMiCoRe extract coupled patterns that capture evolutionary covariation in protein families? (see Section V-E, Section V-F, and Section V-G)

4) Can domain expertise be used to drive the computation of improved alignments? (see Section V-H)

5) How does the experimental run time of ARMiCoRe fare against ClustalW and COBALT (see Section V-I)

\section{A. Datasets}

We use both simulated and benchmark datasets to evaluate our method.

1) Simulated Datasets: To evaluate our proposed method, we designed a simulation model to generate MSAs with embedded coupled patterns. We generated 27 synthetic protein families varying various parameters (see Table I). Subsequently, the multiple sequence alignments were stripped of the gap ('-') symbols to obtain contiguous residue sequences. We used a standard multiple sequence alignment algorithm (in this case ClustalW) to align these sequences and used this new alignment to mine for coupled patterns.

The simulator generates an MSA by first randomly labeling residue positions as either a conserved column, randomly
TABLE I: Description of simulated datasets. Each of the dataset from A0 to $\mathrm{F} 2$ has 100 sequences and 100 residues.

\begin{tabular}{|c|c|l|}
\hline Dataset & Parameter Value & Parameter Description \\
\hline A0-A2 & $\{0.2,0.4,0.6\}$ & $\begin{array}{l}\text { Fraction of columns involved in cou- } \\
\text { plings }\end{array}$ \\
\hline B0-B3 & $\{2,3,4,5\}$ & $\begin{array}{l}\text { Number of columns in each embedded } \\
\text { coupled pattern }\end{array}$ \\
\hline C0-C3 & $\{2,3,4,5\}$ & $\begin{array}{l}\text { Number of partitions or blocks in each } \\
\text { embedded coupled pattern }\end{array}$ \\
\hline D0-D2 & $\{0.2,0.4,0.6\}$ & $\begin{array}{l}\text { Fraction of sequences covered by the } \\
\text { dominant or combination in each cou- } \\
\text { pled pattern }\end{array}$ \\
\hline F0-F2 & $\{0.4,0.6,0.8\}$ & $\begin{array}{l}\text { Fraction of sequences covered by the } \\
\text { conserved symbol in a given conserved } \\
\text { column }\end{array}$ \\
\hline G0-G2 & $\{50,100,150\}$ & $\begin{array}{l}\text { Fraction of deletions (i.e. blanks '-') in } \\
\text { a column } \\
\text { alignment of columns in a simulated }\end{array}$ \\
\hline H0-H2 & $\{50,100,150\}$ & $\begin{array}{l}\text { Number of sequences in a simulated } \\
\text { alignment }\end{array}$ \\
\hline
\end{tabular}

distributed column, or part of a coupled pattern. Each conserved column is then assigned a dominant symbols randomly drawn from 20 amino acid residues and each row of the MSA for that residue position gets the dominant symbol with high probability or one of the remaining amino acid symbols (including a gap) with remaining probability. A residue position labeled as random receives amino acid symbols with equal probability. Next, couplings are embedded over the set of columns allocated for this purpose. Each coupled pattern embedded into the MSA consists of two or more sets of symbols where all sets have the same number of distinct residue symbols. Each set of symbols in a coupled pattern is randomly assigned a sequence in the MSA and the symbols of the set are placed in the respective columns of the MSA assigned to that coupling. The number of columns in a coupled pattern, the number of sets or partitions and the total number of coupled patterns to embed are input by the user. There is also a provision in the simulator to set probabilities of assignment to each of the symbol sets or partitions in a coupling. For example in our simulation, we designate one of the residue sets as the dominant combination which is used in a larger fraction of sequences in the MSA.

2) Benchmark Datasets: We evaluate our method using three well-known benchmark datasets: BaliBase3 [32], OXBench [33], and SABRE [34]. The BaliBase benchmark is created for evaluating both pairwise and MSA algorithms. We use only those alignments from BaliBase that have at least 25 sequences, which yields 48 alignments from three reference sets: RV12, RV20, and RV30. (We chose a threshold of 25 sequences in order to maintain the fidelity of couplings within a sequence family.) For reference set RV20 and RV30 we chose additional threshold of 400 residues for sequence length to reduce the number of alignments in the datasets. The alignments in the reference set RV12 are composed of sequences that are equidistant and have 20-40\% identity. The reference set RV20 contains alignments that are composed of highly divergent orphan sequences. The reference set RV30 contains alignments that are composed of sequence groups each of whom have less than $25 \%$ identity. OXBench has 3 
reference sets and the master set contains 673 alignments that have sequences ranges from 2 to 122 . From the master set, we chose a subset that have at least 25 sequences (yields 20 alignments). SABRE contains 423 alignments that have sequences ranges from 3 to 25 . We choose a subset of 6 sequences that have at least 20 sequences.

Other than these benchmark datasets, we use families of proteins couplings: GPCR, WW, and PDZ. G-protein coupled receptors are a key demonstrator of allosteric communication and serve to transduce extracellular stimuli into intracellular signals [35]. The entire GPCR family is subdivided into 16 subfamilies (alignments). We use 6 alignments from this set, each of whom involve at least 30 sequences: Amine, Rhodopsin, Peptide, Olfactory, Nucleotide, and Prostanoid. The PDZ family has only one alignment and the WW family has three subfamilies: native, CC, and IC.

A summary of the alignments that are used in the experiments are shown in Table II.

\section{B. Scoring Criteria}

We use four different scoring criteria to assess the quality of a test alignment with respect to a reference alignment. The scores are as follows:

1) Q-Score [4]: This score, a.k.a. sum-of-pairs score, can be defined as follows. Let $T$ be the number of aligned residue pairs in the reference alignment and $L$ be the number of aligned residues pairs in the reference alignment that are also correctly aligned in the test alignment. Then, Q-score $=\frac{L}{T}$.

2) Total Column Score (TC) [32]: This score is measured by the percent of the number of columns in the reference alignment that are identical with a test alignment. Let $m$ be the number of columns in a reference alignment and $m^{\prime}$ be the number of columns that are identical in both of the reference and test alignments. Then, TC-Score $=$ $\frac{m^{\prime}}{m}$.

3) Modeler Score [36]: This score is the same as the Qscore but with a different denominator. The score is the percent of pairs of residues in the test alignment that are present in the reference alignment. Let $R$ be the number of aligned residue pairs in a test alignment and $L$ be the number of aligned residue pairs in the reference alignment that are also correctly aligned in the test alignment. Then, Modeler score $=\frac{L}{R}$.

4) Cline Shift Score [37]: While the above three scores evaluate only correctly aligned residues or residue pairs, the Shift score also penalizes misalignments. See [37] for more details.

5) Coupled Column Score (C-Score): None of the above four scores measure how many of the coupled columns (columns that are participating in the couplings) of a reference alignment are retained in the test alignment. We propose a new score to measure the fraction of retained coupled columns based on probabilistic graphical models (PGMs). PGMs can encode couplings of an alignment [38] where each node denotes a column of the alignment and each edge denotes a coupling between
TABLE III: Comparison of ARMiCoRe with ClustalW and Cobalt on synthetic dataset.

\begin{tabular}{|l|l|l|l|}
\hline Score & ClustalW & Cobalt & ARMiCoRe \\
\hline Q-score & 0.516 & 0.265 & $\mathbf{0 . 5 5 1}$ \\
\hline TC Score & 0.0 & 0.009 & $\mathbf{0 . 0 1 7}$ \\
\hline Shift score & 0.655 & 0.355 & $\mathbf{0 . 6 6 3}$ \\
\hline Modeler Score & 0.512 & 0.474 & $\mathbf{0 . 5 9 2}$ \\
\hline
\end{tabular}

two columns. To calculate the C-Score, we create a PGM for a reference alignment, and then count the number of columns $(V)$ that are participating in couplings. For these $V$ coupled columns in the reference alignment, we count how many $\left(V^{\prime}\right)$ of them are retained in the test alignment. A column in the reference alignment is considered to be retained in the test alignment if the number of mismatched residues are fewer than $10 \%$ of the residues in the particular column in the reference alignment. These two counts give us C-Score $=\frac{V^{\prime}}{V}$.

For all the above measures, higher values are better. The five measures yield a maximum score of 1 . The first four measures yield a score of 1 when both the reference and test alignments are identical. The first three measures yield a score of 0 when the alignments are a complete mismatch. For the Shift score, the minimum possible score is -0.2 by default.

\section{Effects of Important Thresholds}

The parameters that have the most significant impact on the number of coupled patterns discovered are the dominant residue conservation threshold $\left(\tau_{d}\right)$, block coverage threshold $(\tau)$, and column-window size threshold $(\epsilon)$. Based on the 27 synthetic alignments, we produced precision-recall curves using various values for $\tau_{d}, \tau$ and $\epsilon$. In synthetic alignments couplings are embedded. We run our methods on these datasets to discover coupled patterns based on various parameters and see how many of the discovered coupled patterns are matched (true positive) and how many are redundant (false positive). The precision-recall curve for $\tau_{d} \in\{0.2,0.4,0.6,0.8\}$ which are illustrated in Fig. 6. We vary the block pattern threshold parameter $\tau$ in the set $\{0.4,0.6,0.8,0.10,0.12\}$ and generate precision-recall plots (Fig. not shown). Similarly, we produce precision-recall curves for $\epsilon \in\{1,2,3,4,5\}$ (Fig. not shown). As all the plots reveal, our method maintain consistently high levels of recall and precision across a wide range of thresholds.

\section{Comparison with ClustalW and COBALT}

We evaluate ARMiCoRe on all the datasets described earlier: synthetic, benchmark and alignments with couplings. For each of these alignments, we remove gaps and realign with ClustalW in default settings (using the PAM matrix). We then run ARMiCoRe on each of the ClustalW alignments to generate coupled patterns and use the coupled patterns to generate constraints, which are used by COBALT to create an improved alignment. We then compare our scores with ClustalW and with COBALT (without any constraints input).

As shown in Table III ARMiCoRe excels in all four traditional measures of MSA quality for synthetic datasets. 
TABLE II: Summary of datasets used in the study.

\begin{tabular}{|l|c|c|c|c|c|}
\hline Dataset & $\begin{array}{l}\text { Num of } \\
\text { Alignments }\end{array}$ & $\begin{array}{l}\text { Min Number } \\
\text { of Sequences }\end{array}$ & $\begin{array}{l}\text { Max Number } \\
\text { of Sequences }\end{array}$ & $\begin{array}{l}\text { Min Number } \\
\text { of Residues }\end{array}$ & $\begin{array}{l}\text { Max Number } \\
\text { of Residues }\end{array}$ \\
\hline Synthetic & 27 & 50 & 150 & 50 & 150 \\
\hline BaliBase(RV12) & 4 & 27 & 34 & 268 & 786 \\
\hline BaliBase(RV20) & 27 & 30 & 91 & 63 & 400 \\
\hline BaliBase(RV30) & 17 & 34 & 140 & 69 & 358 \\
\hline OXBench & 20 & 26 & 122 & 49 & 174 \\
\hline SABRE & 6 & 25 & 25 & 525 & 525 \\
\hline CC & 1 & 43 & 43 & 39 & 39 \\
\hline PDZ & 1 & 80 & 80 & 80 & 80 \\
\hline Nucleotide & 1 & 41 & 41 & 348 & 348 \\
\hline Olfactory & 1 & 41 & 41 & 348 & 348 \\
\hline Prostanoid & 1 & 33 & 33 & 348 & 348 \\
\hline Amine & 1 & 196 & 196 & 348 & 348 \\
\hline Peptide & 1 & 333 & 333 & 348 & 348 \\
\hline Rhodopsin & 1 & 143 & 143 & 348 & 348 \\
\hline
\end{tabular}

(A)

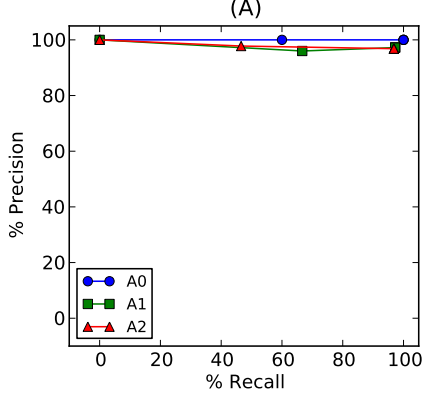

(E)

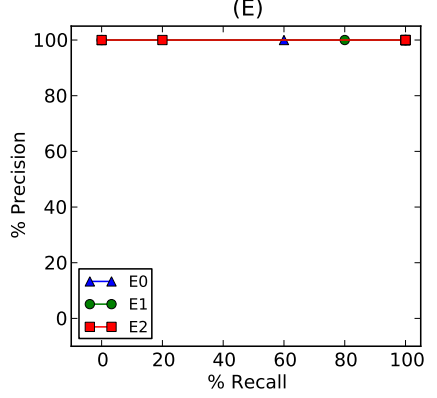

Varying Conservation Threshold from 0.2 to 0.8

(B)

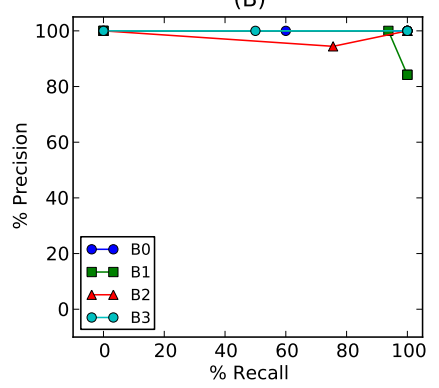

(F)

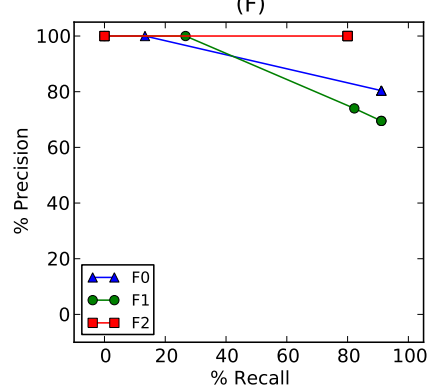

(C)

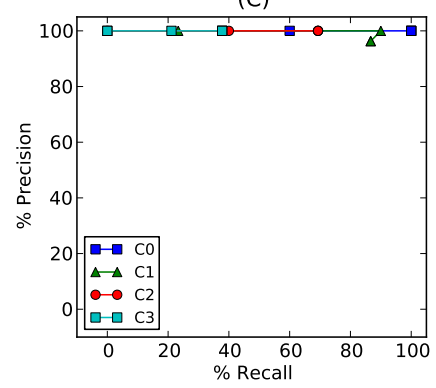

(G)

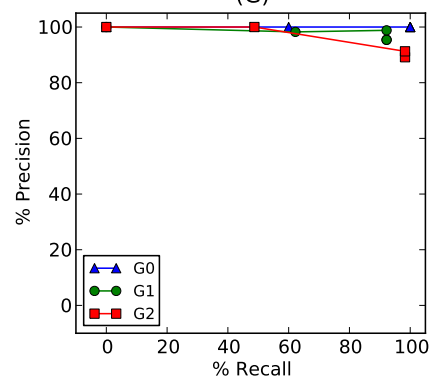

(D)

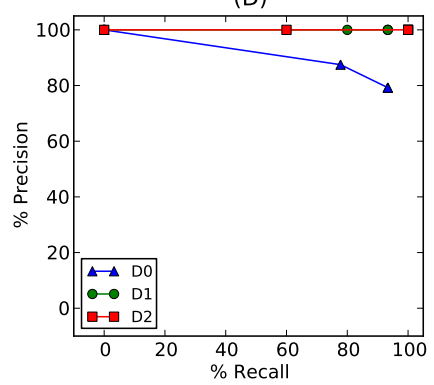

(H)

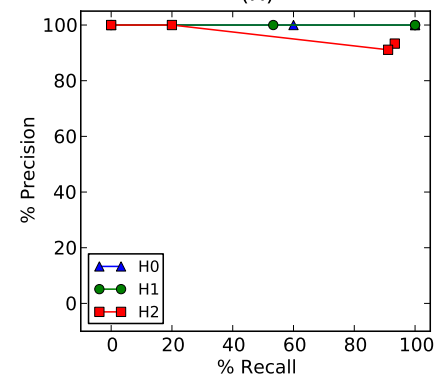

Fig. 6: Precision-recall plots for the dominant residue conservation threshold $\tau_{d}[0.2,0.4,0.6,0.8]$.

TABLE IV: Comparison of ARMiCoRe with Cobalt on RV12 reference set of the Balibase [32] benchmark.

\begin{tabular}{|l|r|r|r|r|r|r|r|r|}
\hline \multirow{2}{*}{ Dataset } & \multicolumn{2}{|c|}{ Q-Score } & \multicolumn{2}{c|}{ TC Score } & \multicolumn{2}{c|}{ Shift Score } & \multicolumn{2}{c|}{ Modeler Score } \\
\cline { 2 - 9 } & Cobalt & ARMiCoRe & Cobalt & ARMiCoRe & Cobalt & ARMiCoRe & Cobalt & ARMiCoRe \\
\hline BB12035 & $\mathbf{0 . 7 5}$ & 0.74 & 0.20 & $\mathbf{0 . 2 5}$ & $\mathbf{0 . 8 1}$ & 0.80 & $\mathbf{0 . 8 4}$ & 0.78 \\
\hline BB12043 & $\mathbf{0 . 6 8}$ & 0.66 & $\mathbf{0 . 1 0}$ & $\mathbf{0 . 1 0}$ & $\mathbf{0 . 7 7}$ & 0.72 & $\mathbf{0 . 7 8}$ & 0.72 \\
\hline BBS12035 & 0.78 & $\mathbf{0 . 8 1}$ & 0.30 & $\mathbf{0 . 3 8}$ & 0.84 & $\mathbf{0 . 8 6}$ & $\mathbf{0 . 8 6}$ & 0.83 \\
\hline BBS12043 & 0.75 & $\mathbf{0 . 8 0}$ & 0.24 & $\mathbf{0 . 3 3}$ & 0.82 & $\mathbf{0 . 8 5}$ & $\mathbf{0 . 8 4}$ & 0.82 \\
\hline Avg & 0.74 & $\mathbf{0 . 7 5}$ & 0.21 & $\mathbf{0 . 2 6}$ & $\mathbf{0 . 8 1}$ & $\mathbf{0 . 8 1}$ & $\mathbf{0 . 8 3}$ & 0.79 \\
\hline
\end{tabular}

TABLE V: Comparison of ARMiCoRe against ClustalW over all BaliBase datasets (using only core regions). The average scores are shown here. RV20* is curated from RV20 by removing orphan sequences.

\begin{tabular}{|l|l|r|r|r|r|r|r|r|}
\hline \multirow{2}{*}{ Dataset } & \multicolumn{3}{|c|}{ Q-Score } & \multicolumn{2}{c|}{ TC Score } & \multicolumn{2}{c|}{ Shift Score } & \multicolumn{2}{c|}{ Modeler Score } \\
\cline { 2 - 9 } & ClustalW & ARMiCoRe & ClustalW & ARMiCoRe & ClustalW & ARMiCoRe & ClustalW & ARMiCoRe \\
\hline RV12 & 0.84 & $\mathbf{0 . 8 9}$ & 0.51 & $\mathbf{0 . 6 1}$ & 0.73 & $\mathbf{0 . 8 1}$ & 0.69 & $\mathbf{0 . 7 9}$ \\
\hline RV20 & $\mathbf{0 . 8 4}$ & 0.79 & $\mathbf{0 . 2 4}$ & 0.15 & $\mathbf{0 . 8 3}$ & 0.78 & $\mathbf{0 . 8 1}$ & 0.78 \\
\hline RV20 & 0.88 & 0.90 & 0.54 & $\mathbf{0 . 5 7}$ & $\mathbf{0 . 8 8}$ & $\mathbf{0 . 8 8}$ & 0.85 & $\mathbf{0 . 8 7}$ \\
\hline RV30 & $\mathbf{0 . 6 8}$ & 0.58 & $\mathbf{0 . 2 3}$ & 0.13 & $\mathbf{0 . 6 5}$ & 0.58 & $\mathbf{0 . 6 3}$ & $\mathbf{0 . 6 3}$ \\
\hline
\end{tabular}


TABLE VI: Comparison of ARMiCoRe against ClustalW and COBALT over OXBench alignments.

\begin{tabular}{|c|c|c|c|c|}
\hline Dataset & Score & ClustalW & COBALT & ARMiCoRe \\
\hline \multirow{4}{*}{$12 \mathrm{~s} 107$} & Q-Score & 0.99 & 0.99 & 0.98 \\
\hline & TC Score & 0.80 & 0.93 & 0.86 \\
\hline & Shift Score & 0.93 & 0.93 & 0.92 \\
\hline & Modeler Score & 0.87 & 0.87 & 0.87 \\
\hline \multirow{4}{*}{$12 \mathrm{~s} 108$} & Q-Score & 0.97 & 0.99 & 0.99 \\
\hline & TC Score & 0.85 & 0.97 & 0.94 \\
\hline & Shift Score & 0.88 & 0.89 & 0.89 \\
\hline & Modeler Score & 0.79 & 0.81 & 0.81 \\
\hline \multirow{4}{*}{$12 \mathrm{t} 109$} & Q-Score & 0.96 & 0.99 & 0.96 \\
\hline & TC Score & 0.76 & 0.91 & 0.8 \\
\hline & Shift Score & 0.87 & 0.89 & 0.87 \\
\hline & Modeler Score & 0.78 & 0.80 & 0.80 \\
\hline \multirow{4}{*}{$12 \mathrm{t} 113$} & Q-Score & 0.95 & 0.99 & 0.91 \\
\hline & TC Score & 0.82 & 0.92 & 0.56 \\
\hline & Shift Score & 0.78 & 0.80 & 0.76 \\
\hline & Modeler Score & 0.65 & 0.68 & 0.67 \\
\hline \multirow{4}{*}{$12 \mathrm{t} 116$} & Q-Score & 0.94 & 0.98 & 0.87 \\
\hline & TC Score & 0.53 & 0.65 & 0.33 \\
\hline & Shift Score & 0.76 & 0.79 & 0.73 \\
\hline & Modeler Score & 0.62 & 0.64 & 0.65 \\
\hline$\vdots$ & $\vdots$ & : & : & : \\
\hline \multirow{4}{*}{$588 \mathrm{t} 28$} & Q-Score & 0.99 & 0.98 & 0.99 \\
\hline & TC Score & 0.97 & 0.94 & 0.97 \\
\hline & Shift Score & 0.89 & 0.88 & 0.89 \\
\hline & Modeler Score & 0.80 & 0.80 & 0.79 \\
\hline \multirow{4}{*}{ _22s38 } & Q-Score & 0.95 & 0.95 & 0.95 \\
\hline & TC Score & 0.82 & 0.81 & 0.81 \\
\hline & Shift Score & 0.81 & 0.81 & 0.81 \\
\hline & Modeler Score & 0.69 & 0.69 & 0.69 \\
\hline \multirow{4}{*}{$-22 \mathrm{t} 50$} & Q-Score & 0.96 & 0.95 & 0.95 \\
\hline & TC Score & 0.86 & 0.83 & 0.85 \\
\hline & Shift Score & 0.78 & 0.77 & 0.78 \\
\hline & Modeler Score & 0.64 & 0.64 & 0.63 \\
\hline \multirow{4}{*}{588} & Q-Score & 0.98 & 0.99 & 0.98 \\
\hline & TC Score & 0.83 & 0.83 & 0.8 \\
\hline & Shift Score & 0.88 & 0.89 & 0.89 \\
\hline & Modeler Score & 0.80 & 0.80 & 0.80 \\
\hline \multirow{4}{*}{12} & Q-Score & 0.86 & 0.88 & 0.87 \\
\hline & TC Score & 0.00 & 0.00 & 0.1 \\
\hline & Shift Score & 0.50 & 0.51 & 0.53 \\
\hline & Modeler Score & 0.34 & 0.35 & 0.36 \\
\hline
\end{tabular}

Table IV depicts the superior performance of ARMiCoRe over COBALT on three of the four measures in the RV12 reference set of the BaliBase benchmark. Performance of ARMiCoRe against ClustalW on all reference sets of the BaliBase benchmark is given in Table V. ARMiCoRe shows superior performance over ClustalW on all of the four measures in the RV12 reference set. The sequence identity in this benchmark is about $20-40 \%$. Note that the performance of ARMiCoRe on RV20 and RV30 is worse than that of ClustalW in all four measures. This is because RV20 and RV30 pool together sequences with poor similarity and thus coupled patterns are not a driver for obtaining good alignments. The effect of an orphan sequence on the similarity structure of an alignment is illustrated in Fig. 7. To test this hypothesis, we removed the orphan sequences from RV20 (RV20*) and as Table $\mathrm{V}$ shows, the performance of ARMiCoRe is better along three of the four measures. Table VI describes the results of ARMiCoRe for the OXBench benchmark, once again revealing a mixed performance on a dataset with high sequence diversity. Finally, Table VII depicts the superior per-
TABLE VII: Comparison of ARMiCoRe against ClustalW and COBALT over SABRE alignments.

\begin{tabular}{|c|c|c|c|c|}
\hline Dataset & Score & ClustalW & COBALT & ARMiCoRe \\
\hline \multirow{4}{*}{ sup_038 } & Q-Score & 0.82 & 0.83 & 0.88 \\
\hline & TC Score & 0 & 0 & 0 \\
\hline & Shift Score & 0.17 & 0.18 & 0.19 \\
\hline & Modeler Score & 0.10 & 0.10 & 0.12 \\
\hline \multirow{4}{*}{ sup_092 } & Q-Score & 0.20 & 0.28 & 0.30 \\
\hline & TC Score & 0 & 0 & 0 \\
\hline & Shift Score & 0.05 & 0.09 & 0.07 \\
\hline & Modeler Score & 0.03 & 0.05 & 0.05 \\
\hline \multirow{4}{*}{ sup_108 } & Q-Score & 0.89 & 0.92 & 0.93 \\
\hline & TC Score & 0 & 0.35 & 0.35 \\
\hline & Shift Score & 0.46 & 0.48 & 0.48 \\
\hline & Modeler Score & 0.31 & 0.32 & 0.32 \\
\hline \multirow{4}{*}{ sup_126 } & Q-Score & 0.51 & 0.76 & 0.59 \\
\hline & TC Score & 0 & 0.14 & 0 \\
\hline & Shift Score & 0.19 & 0.30 & 0.23 \\
\hline & Modeler Score & 0.12 & 0.18 & 0.14 \\
\hline \multirow{4}{*}{ sup_167 } & Q-Score & 0.61 & 0.69 & 0.50 \\
\hline & TC Score & 0 & 0 & 0 \\
\hline & Shift Score & 0.27 & 0.33 & 0.23 \\
\hline & Modeler Score & 0.17 & 0.21 & 0.14 \\
\hline \multirow{4}{*}{ sup_215 } & Q-Score & 0.11 & 0.47 & 0.18 \\
\hline & TC Score & 0 & 0 & 0 \\
\hline & Shift Score & 0.0 & 0.03 & 0.01 \\
\hline & Modeler Score & 0.0 & 0.01 & 0.01 \\
\hline
\end{tabular}

TABLE VIII: Comparison of ARMiCoRe against ClustalW and COBALT over CC subfamily of WW protein family, PDZ family, and Nucleotide subfamily of GPCR family.

\begin{tabular}{|c|l|r|r|r|}
\hline Dataset & Score & ClustalW & COBALT & ARMiCoRe \\
\hline \multirow{5}{*}{ CC } & & & & \\
\cline { 2 - 5 } & Q-Score & 0.89 & 0.85 & $\mathbf{0 . 9 6}$ \\
\cline { 2 - 5 } & TC Score & $\mathbf{0 . 5 1}$ & 0.35 & $\mathbf{0 . 5 1}$ \\
\cline { 2 - 5 } & Shift Score & 0.93 & 0.91 & $\mathbf{0 . 9 7}$ \\
\cline { 2 - 5 } & Modeler Score & 0.90 & 0.91 & $\mathbf{0 . 9 7}$ \\
\cline { 2 - 5 } & C-Score & 0.71 & 0.88 & $\mathbf{1 . 0 0}$ \\
\hline \multirow{5}{*}{ PDZ } & Q-Score & 0.85 & 0.82 & $\mathbf{0 . 8 7}$ \\
\cline { 2 - 5 } & TC Score & 0 & $\mathbf{0 . 1}$ & 0 \\
\cline { 2 - 5 } & Shift Score & 0.89 & 0.87 & $\mathbf{0 . 9}$ \\
\cline { 2 - 5 } & Modeler Score & 0.85 & $\mathbf{0 . 8 9}$ & 0.88 \\
\cline { 2 - 5 } & C-Score & 0.67 & $\mathbf{0 . 8 1}$ & $\mathbf{0 . 8 1}$ \\
\hline & Q-Score & 0.74 & 0.68 & $\mathbf{0 . 7 9}$ \\
\cline { 2 - 5 } & TC Score & $\mathbf{0 . 4 6}$ & 0.34 & 0.45 \\
\cline { 2 - 5 } & Shift Score & 0.79 & 0.74 & $\mathbf{0 . 8 3}$ \\
\cline { 2 - 5 } & Modeler Score & 0.74 & 0.77 & $\mathbf{0 . 8 0}$ \\
\cline { 2 - 5 } & C-Score & 0.52 & 0.50 & $\mathbf{0 . 6 3}$ \\
\hline
\end{tabular}

formance of ARMiCoRe over ClustalW in 5 alignments out of 6 alignments in SABRE dataset. In Tables VI and VII, we see that sometimes ARMiCoRe performs relatively poor compare to COBALT. The reason might be the sequence similarity structure of the alignments or poor choice of gap penalties for realignment. Note that these benchmark datasets are not designed for evaluating couplings' discovery algorithms in protein families.

\section{E. Modeling Correlated Mutations}

We describe the effect of ARMiCoRe on three families that are known to exhibit correlated mutations. We focus on the CC subfamily of the WW domain, the PDZ family, and the Nucleotide subfamily of the GPCR family. Based on CScore, we evaluate the Performance of ARMiCoRe against ClustalW and COBALT. As shown in Table VIII, ARMiCoRe is consistently better on at least three measures. 


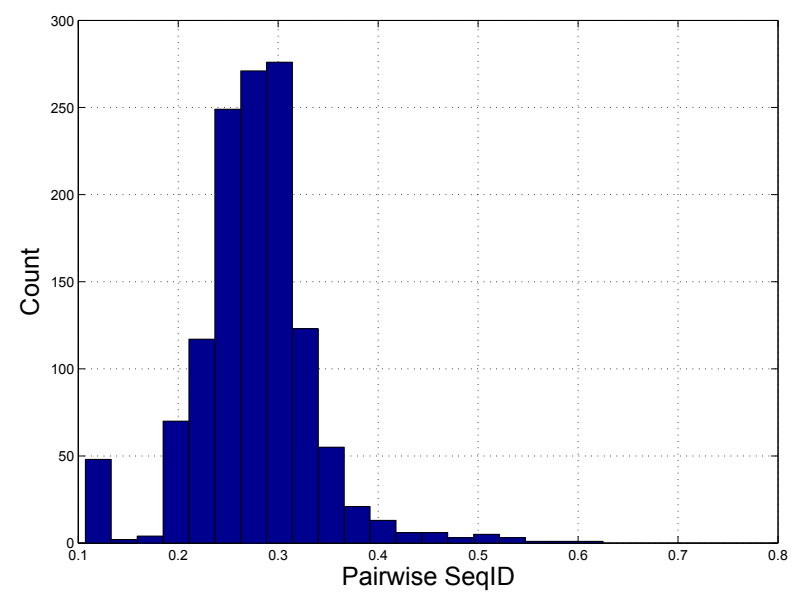

(a)

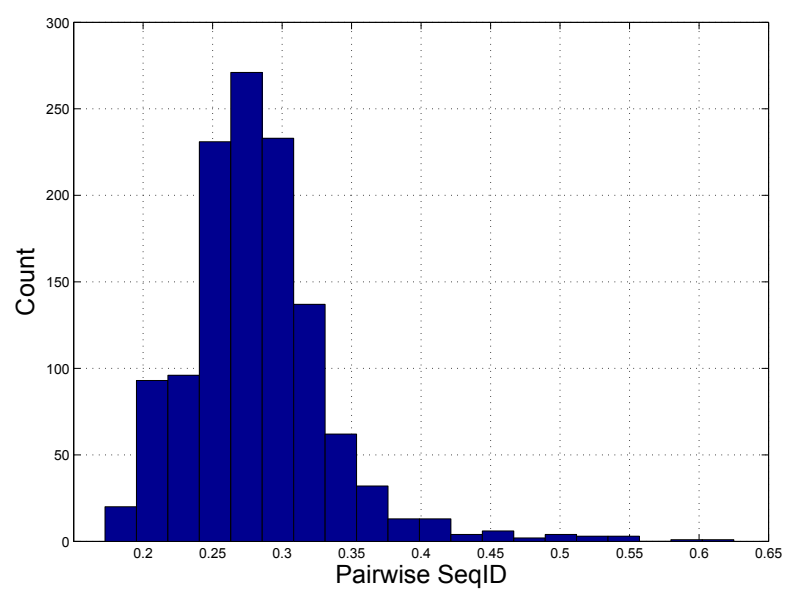

(b)

Fig. 7: Pairwise sequence similarity analysis of an alignment 'BB20006' from RV20 dataset that contains an orphan sequence. We use SCA [39] for this analysis. Fig. 7a has a peak for similarity score around 0.12 that indicates that the orphan is distant from the other sequences. Fig. 7b shows a reasonably narrow distribution without the orphan sequence with a mean pairwise similarity between sequences of about $27 \%$ and a range of $20 \%$ to $35 \%$, which suggests that most sequences are about equally dissimilar from other.

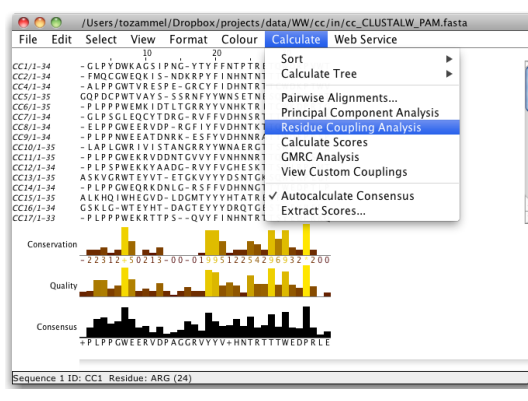

(a)

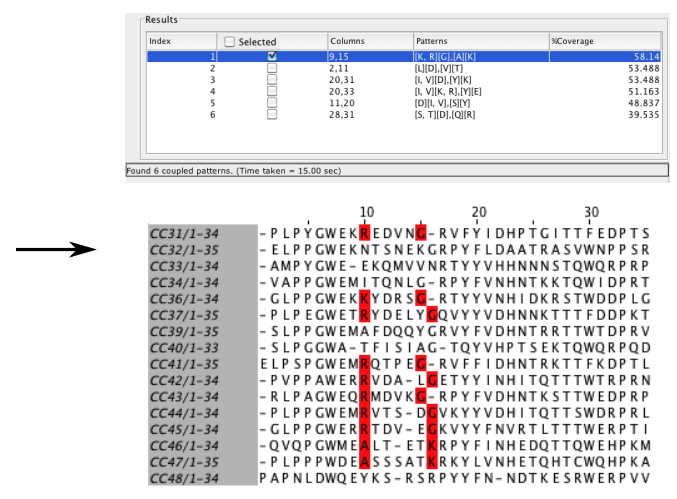

(b)

Fig. 9: Interfaces for mining coupled patterns. (a) Loading of An input alignment. (b) Selection of coupled patterns with colored plot of corresponding residues.

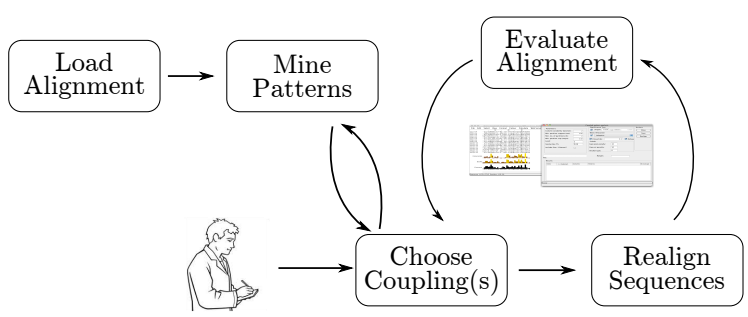

Fig. 8: An overview of user interaction with ARMiCoRe.

\section{F. Evaluation using Global Statistical Model for Residue Couplings}

Couplings are often employed to predict 3D structure of proteins from sequences. A global statistical method for residue couplings for predicting $3 \mathrm{D}$ structure of proteins is proposed by Marks et al. [40]. The proposed method first calculates pairwise coupling scores and then uses high scoring pairs to find a 3D structure. The way of calculating couplings scores is global which is different from the method given by Thomas et al. [38]. We use their method to calculate pairwise couplings scores for reference, ClustalW, and ARMiCoRe alignments. We then identify how many of the coupled pairs (true positive) for the reference alignment are also retained in ClustalW and ARMiCoRe alignments for various thresholds. In Table IX and Table XI we see that the alignments for CC and Nucleotide subfamily given by ARMiCoRe is much better than that of ClustalW. But the alignment for PDZ family given by ARMiCoRe is not better than that of ClustalW in terms of couplings calculated in global settings (see Table X). The scores produced by ARMiCoRe are low for both PDZ and Nucleotide families. To get an insight we perform sequence similarity analysis using SCA tool [39]. We have seen wider distributions of sequence similarities with multiple peaks for PDZ and Nucleotide families which indicates that sequences are not equally dissimilar from each other and the presence of subclusters of sequences within the family. 
TABLE IX: Comparison of ARMiCoRe against ClustalW over the CC subfamily of WW family using the global residue coupling model defined in [40]. Here 'TP' is used for true positive, ' $\mathrm{P}$ ' is used for precision, and ' $\mathrm{R}$ ' is used for recall.

\begin{tabular}{|c|c|c|c|c|c|c|c|c|c|c|c|}
\hline $\begin{array}{l}\text { Score } \\
\text { Threshold }\end{array}$ & $\begin{array}{l}\text { Number of } \\
\text { couplings in } \\
\text { Ref MSA }\end{array}$ & $\begin{array}{l}\text { Number of } \\
\text { couplings in } \\
\text { ClustalW MSA }\end{array}$ & TP & $\mathrm{P}$ & $\mathrm{R}$ & $\begin{array}{l}\mathrm{F} 1 \\
\text { Score }\end{array}$ & $\begin{array}{l}\text { Num of couplings } \\
\text { in ARMiCoRe } \\
\text { MSA }\end{array}$ & $\mathrm{TP}$ & $\mathrm{P}$ & $\mathrm{R}$ & $\begin{array}{l}\text { F1 } \\
\text { Score }\end{array}$ \\
\hline 0.40 & 8 & 5 & 1 & 0.20 & 0.13 & 0.15 & 5 & 3 & 0.60 & 0.38 & $\mathbf{0 . 4 6}$ \\
\hline 0.35 & 27 & 19 & 13 & 0.68 & 0.48 & 0.57 & 29 & 21 & 0.72 & 0.78 & $\mathbf{0 . 7 5}$ \\
\hline 0.30 & 64 & 51 & 36 & 0.71 & 0.56 & 0.63 & 61 & 53 & 0.87 & 0.83 & $\mathbf{0 . 8 5}$ \\
\hline
\end{tabular}

TABLE X: Comparison of ARMiCoRe against ClustalW over the PDZ family using the global residue coupling model defined in [40]. Here 'TP' is used for true positive, ' $P$ ' is used for precision, and ' $R$ ' is used for recall.

\begin{tabular}{|c|l|l|c|c|c|c|c|c|c|c|c|}
\hline $\begin{array}{l}\text { Score } \\
\text { Threshold }\end{array}$ & $\begin{array}{l}\text { Number of } \\
\text { couplings in } \\
\text { Ref MSA }\end{array}$ & $\begin{array}{l}\text { Number of } \\
\text { couplings in } \\
\text { ClustalW MSA }\end{array}$ & TP & $\mathrm{P}$ & $\mathrm{R}$ & $\begin{array}{l}\text { F1 } \\
\text { Score }\end{array}$ & $\begin{array}{l}\text { Num of couplings } \\
\text { in ARMiCoRe } \\
\text { MSA }\end{array}$ & TP & P & R & $\begin{array}{l}\text { F1 } \\
\text { Score }\end{array}$ \\
\hline 0.20 & 15 & 13 & 4 & 0.27 & 0.31 & $\mathbf{0 . 2 9}$ & 17 & 3 & 0.20 & 0.18 & 0.19 \\
\hline 0.15 & 64 & 71 & 21 & 0.33 & 0.30 & $\mathbf{0 . 3 1}$ & 82 & 13 & 0.20 & 0.16 & 0.18 \\
\hline 0.10 & 396 & 405 & 190 & 0.48 & 0.47 & $\mathbf{0 . 4 7}$ & 474 & 145 & 0.37 & 0.31 & 0.33 \\
\hline
\end{tabular}

TABLE XI: Comparison of ARMiCoRe against ClustalW over the Nucleotide subfamily of GPCR family using the global residue coupling model defined in [40]. Here 'TP' is used for true positive, ' $P$ ' is used for precision, and ' $R$ ' is used for recall.

\begin{tabular}{|c|c|c|c|c|c|c|c|c|c|c|c|}
\hline $\begin{array}{l}\text { Score } \\
\text { Threshold }\end{array}$ & $\begin{array}{l}\text { Number of } \\
\text { couplings in } \\
\text { Ref MSA }\end{array}$ & $\begin{array}{l}\text { Number of } \\
\text { couplings in } \\
\text { ClustalW MSA }\end{array}$ & TP & P & R & $\begin{array}{l}\text { F1 } \\
\text { Score }\end{array}$ & $\begin{array}{l}\text { Num of couplings } \\
\text { i ARMiCoRe } \\
\text { MSA }\end{array}$ & TP & P & R & $\begin{array}{l}\text { F1 } \\
\text { Score }\end{array}$ \\
\hline 0.15 & 7 & 7 & 0 & 0.00 & 0.00 & - & 9 & 1 & 0.11 & 0.14 & $\mathbf{0 . 1 3}$ \\
\hline 0.12 & 14 & 14 & 1 & 0.07 & 0.07 & 0.07 & 24 & 3 & 0.13 & 0.21 & $\mathbf{0 . 1 6}$ \\
\hline 0.10 & 21 & 23 & 2 & 0.09 & 0.10 & 0.09 & 37 & 5 & 0.14 & 0.24 & $\mathbf{0 . 1 7}$ \\
\hline
\end{tabular}

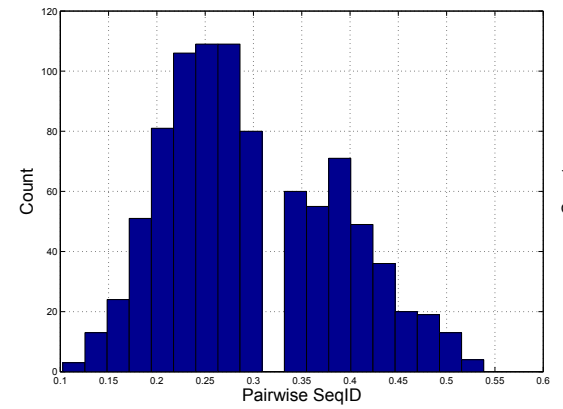

(a)

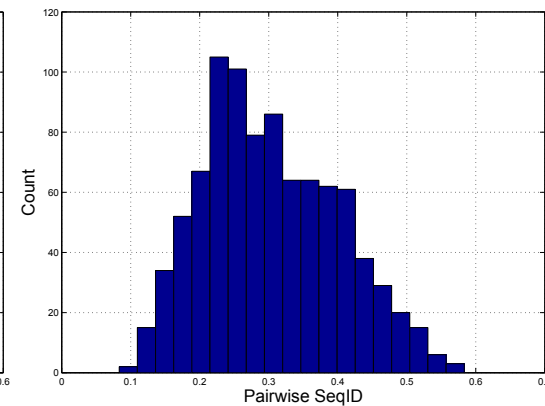

(b)

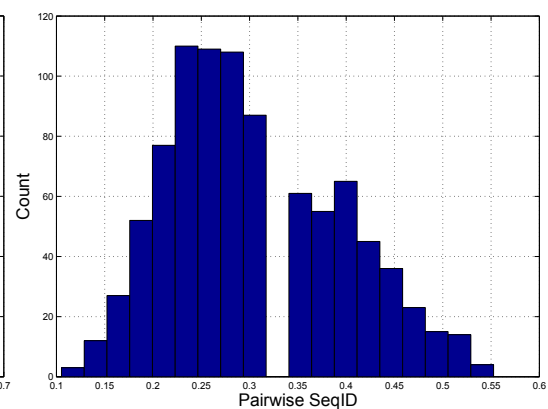

(c)

Fig. 10: Pairwise sequence similarity analysis using SCA [39]. Histograms for reference, ClustalW, and ARMiCoRe are drawn for the same number of bins. This figure shows that the ARMiCoRe alignment retains most of the sequence similarity structure of the reference alignment.

TABLE XII: Comparison of ARMiCoRe against ClustalW over the CC subfamily of WW family using the statistical coupling analysis defined in [41]. Here 'TP' is used for true positive, ' $\mathrm{P}$ ' is used for precision, and ' $\mathrm{R}$ ' is used for recall.

\begin{tabular}{|c|c|c|c|c|c|c|c|c|c|c|c|}
\hline $\begin{array}{l}\text { Cut-off } \\
\text { Threshold }\end{array}$ & $\begin{array}{l}\text { Sector Size } \\
\text { in Ref MSA }\end{array}$ & $\begin{array}{l}\text { Sector Size in } \\
\text { ClustalW MSA }\end{array}$ & TP & $\mathrm{P}$ & $\mathrm{R}$ & $\begin{array}{l}\text { F1 } \\
\text { Score }\end{array}$ & $\begin{array}{l}\text { Sector Size in } \\
\text { ARMiCoRe MSA }\end{array}$ & TP & $\mathrm{P}$ & $\mathrm{R}$ & $\begin{array}{l}\text { F1 } \\
\text { Score }\end{array}$ \\
\hline 0.85 & 7 & 5 & 5 & 1.00 & 0.71 & 0.83 & 7 & 6 & 0.86 & 0.86 & $\mathbf{0 . 8 6}$ \\
\hline 0.80 & 11 & 7 & 7 & 1.00 & 0.64 & 0.78 & 9 & 9 & 1.00 & 0.82 & $\mathbf{0 . 9 0}$ \\
\hline 0.75 & 12 & 8 & 8 & 1.00 & 0.67 & 0.80 & 12 & 11 & 0.92 & 0.92 & $\mathbf{0 . 9 2}$ \\
\hline
\end{tabular}

\section{G. Evaluation using Statistical Coupling Analysis}

Lockless and Ranganathan [41] proposed statistical coupling analysis (SCA) as a method for analyzing coevolution in protein families represented by MSAs. The SCA tool [39] performs sequence similarity analysis to get an idea of the number of subfamilies in the MSA. We perform similarity analysis for the reference, ClustalW, and ARMiCoRe alignments of CC family (see Fig. 10). There are two subfamilies for the CC family: folded and non-folded. Fig. 10 shows that for both reference and ARMiCoRe alignments there are two peaks in the histogram which is an indication that there are two subfamilies, whereas the ClustalW alignment indicates that the similarity structure for two subfamilies are distorted.

SCA also allows us to identify protein sectors, which are quasi-independent groups of correlated amino acids [42]. We identify protein sectors of reference, ClustalW, and ARMiCoRe alignments for various cut-off thresholds $(0.85,0.80$, and 0.75$)$. We then calculate precision, recall, and F1-score 
TABLE XIII: Comparison of ARMiCoRe with ClustalW and COBALT using avergare runtime per alignment. Times are given in seconds.

\begin{tabular}{|l|c|c|c|}
\hline Dataset & ClustalW & COBALT & ARMiCoRe \\
\hline BaliBase & 3.2 & $\mathbf{1 . 7 4}$ & 5.23 \\
\hline OXBench & 2.76 & $\mathbf{2 . 1 8}$ & 4.77 \\
\hline SABRE & $\mathbf{0 . 8 0}$ & 3.41 & 3.53 \\
\hline
\end{tabular}

for ClustalW and ARMiCoRe alignments with respect to the reference alignment. For all of the cut-off thresholds one protein sector is identified. Table XII shows that much of protein sector in the reference alignment is retained in the ARMiCoRe alignment.

\section{H. User Interaction in Choosing Couplings}

We have developed GUIs for ARMiCoRe that allow users to interactively choose patterns from a set of significant coupled patterns and use them to realign sequences. This enables biologists to bring specific domain knowledge in deciding which coupled pattern sets should be exposed as couplings in the new alignment. We have integrated ARMiCoRe with the JalView [43] framework, which has a rich set of sequence analysis tools. A typical workflow with ARMiCoRe is illustrated in Fig. 8. A user begins an experiment by loading an initial alignment (see Fig. 9(a)). He or she can evaluate the input alignment by measuring various scores with respect to a reference alignment. Based on the evaluation, he or she may decide to improve the alignment using the coupled pattern mining module. The coupled pattern mining module facilitates tuning various parameters prior to the pattern mining and gives a set of significant coupled patterns as output. From the pool of coupled patterns, a domain expert can choose meaningful patterns (see Fig. 9(b)) and use them in the realignment module. The realignment module gives a new alignment, which can be evaluated in the evaluation module. A user may repeat the realignment step by choosing different patterns or the mining step by tuning the parameters.

\section{Experimental Runtime}

We compare ARMiCoRe against ClustalW and COBALT based on experimental runtime on three benchmark datasets: BaliBase, OXBench, and SABRE (see Table XIII). As expected ARMiCoRe takes more time than ClustalW and COBALT but the runtime of ARMiCoRe is reasonable.

\section{DISCUSSION}

Evolutionary constraints on genes and proteins to maintain structure and function are revealed as conservation and coupling in an MSA. The advent of cheap, high-throughput sequencing promises to provide a wealth of sequence data enabling such applications, but at the same time requires methods such as ARMiCoRe to improve the alignments and inferred constraints upon which they are based. The alignments obtained by ARMiCoRe can be leveraged to design or classify novel proteins that are stably folded and functional [44], [45], [41], as well as to predict three-dimensional structures from sequence alone [40], [46]. Our work also demonstrates a successful application of pattern set mining where the goal is not just to find patterns but to cover the set of sequences with discovered patterns such that an objective measure is optimized. The ideas developed here can be generalized to other pattern set mining problems in areas like neuroscience, sustainability, and systems biology.

\section{ACKNOWLEDGEMENT}

This work is supported in part by US NSF grant IIS0905313.

\section{REFERENCES}

[1] T. Hubbard, A. Lesk, and A. Tramontano, "Gathering Them in to the Fold," Nat. Struct. Mol. Biol., vol. 3, no. 4, p. 313, 1996.

[2] J. Thompson, D. Higgins, and T. Gibson, "CLUSTAL W: Improving the Sensitivity of Progressive Multiple Sequence Alignment through Sequence Weighting, Position-specific Gap Penalties and Weight Matrix Choice," Nucleic Acids Res., vol. 22, pp. 4673-4680, 1994.

[3] A. Löytynoja and M. Milinkovitch, "A Hidden Markov Model for Progressive Multiple Alignment," Bioinformatics, vol. 19, no. 12, pp. 1505-1513, 2003.

[4] R. Edgar, "MUSCLE: Multiple Sequence Alignment with High Accuracy and High Throughput," Nucleic Acids Res., vol. 32, pp. 1792-1797, 2004.

[5] C. Notredame, D. Higgins, and J. Heringa, "T-coffee: A Novel Method for Fast and Accurate Multiple Sequence Alignment," J. Mol. Biol., vol. 302, no. 1, pp. 205-217, 2000.

[6] B. Bringmann, S. Nijssen, N. Tatti, J. Vreeken, and A. Zimmermann. Mining Sets of Patterns: A Tutorial at ECMLPKDD 2010, Barcelona, Spain. [Online]. Available: http://www.cs.kuleuven.be/conference/msop/

[7] R. Edgar and S. Batzoglou, "Multiple Sequence Alignment," Curr. Opin. Struct. Biol., vol. 16, no. 3, pp. 368-373, 2006.

[8] C. Do and K. Katoh, "Protein Multiple Sequence Alignment," in Functional Proteomics, 2008, vol. 484, ch. 25, pp. 379-413.

[9] K. Katoh, K. Misawa, K. Kuma, and T. Miyata, "MAFFT: A Novel Method for Rapid Multiple Sequence Alignment Based on Fast Fourier Transform," Nucleic Acids Res., vol. 30, no. 14, pp. 3059-3066, 2002.

[10] C. Do, M. Mahabhashyam, M. Brudno, and S. Batzoglou, "ProbCons: Probabilistic Consistency-based Multiple Sequence Alignment," Genome Res., vol. 15, pp. 330-340, 2005.

[11] B. Morgenstern, "DIALIGN: Multiple DNA and Protein Sequence Alignment at BiBiServ," Nucleic Acids Res., vol. 32, no. suppl 2, pp. W33-W36, 2004.

[12] A. Subramanian, J. Weyer-Menkhoff, M. Kaufmann, and B. Morgenstern, "DIALIGN-T: An Improved Algorithm for Segment-based Multiple Sequence Alignment," BMC Bioinformatics, vol. 6, no. 1, p. 66, 2005.

[13] C. Lee, C. Grasso, and M. Sharlow, "Multiple Sequence Alignment using Partial Order Graphs," Bioinformatics, vol. 18, no. 3, pp. 452-464, 2002.

[14] H. Carrillo and D. Lipman, "The Multiple Sequence Alignment Problem in Biology," SIAM J. Appl. Math., vol. 48, no. 5, pp. 1073-1082, 1988.

[15] L. Wang and T. Jiang, "On the Complexity of Multiple Sequence Alignment," J. Comp. Biol., vol. 1, no. 4, pp. 337-348, 1994.

[16] M. Brudno, C. B. Do, G. M. Cooper, M. F. Kim, E. Davydov, E. D. Green, A. Sidow, S. Batzoglou et al., "LAGAN and Multi-LAGAN: Efficient Tools for Large-Scale Multiple Alignment of Genomic DNA," Genome Res., vol. 13, no. 4, pp. 721-731, 2003.

[17] S. Yamada, O. Gotoh, and H. Yamana, "Improvement in Accuracy of Multiple Sequence Alignment using Novel Group-to-group Sequence Alignment Algorithm with Piecewise Linear Gap Cost," BMC Bioinformatics, vol. 7, no. 1, p. 524, 2006.

[18] O. Gotoh, "Significant Improvement in Accuracy of Multiple Protein Sequence Alignments by Iterative Refinement as Assessed by Reference to Structural Alignments," J. Mol. Biol., vol. 264, no. 4, pp. 823-838, 1996.

[19] W. Bains, "MULTAN: A Program to Align Multiple DNA Sequences," Nucleic Acids Res., vol. 14, no. 1, pp. 159-177, 1986.

[20] J. Pei and N. Grishin, "PROMALS: Towards Accurate Multiple Sequence Alignments of Distantly Related Proteins," Bioinformatics, vol. 23, no. 7, pp. 802-808, 2007. 
[21] J. Pei, R. Sadreyev, and N. Grishin, "PCMA: Fast and Accurate Multiple Sequence Alignment Based on Profile Consistency," Bioinformatics, vol. 19 , no. 3, pp. 427-428, 2003.

[22] J. Papadopoulos and R. Agarwala, "COBALT: Constraint-based Alignment Tool for Multiple Protein Sequences," Bioinformatics, vol. 23, no. 9, pp. 1073-1079, 2007.

[23] J. D. Thompson, F. Plewniak, J. Thierry, and O. Poch, "DbClustal: Rapid and Reliable Global Multiple Alignments of Protein Sequences Detected by Database Searches," Nucleic Acids Res., vol. 28, no. 15, pp. 2919 2926, 2000

[24] L. Guasco, "Multiple Sequence Alignment Correction using Constraints," Ph.D. dissertation, Universidade Nova de Lisboa, 2010.

[25] R. Gouveia-Oliveira and A. Pedersen, "Finding Coevolving Amino Acid Residues using Row and Column Weighting of Mutual Information and Multi-dimensional Amino Acid Representation," Algorithms for Molecular Biology, vol. 1, p. 12, 2007.

[26] R. Agrawal, H. Mannila, R. Srikant, H. Toivonen, and A. Verkamo, "Fast discovery of association rules," Advances in Knowledge Discovery and Data Mining, pp. 307-328, 1996.

[27] W. Taylor, "The Classification of Amino Acid Conservation," J. Theor. Biol., vol. 119, pp. 205-218, 1986.

[28] G. Nemhauser, L. Wolsey, and M. Fisher, "An Analysis of Approximations for Maximizing Submodular Set Functions-I," Math. Prog., vol. 14 pp. 265-294, 1978.

[29] J. Edmonds and R. M. Karp, "Theoretical Improvements in Algorithmic Efficiency for Network Flow Problems," J. ACM, vol. 19, pp. 248-264, April 1972.

[30] A. Goldberg and R. Tarjan, "A New Approach to the Maximum-Flow Problem," J. ACM, vol. 35, pp. 921-940, 1988.

[31] B. Morgenstern, K. Frech, A. Dress, and T. Werner, "DIALIGN: Finding Local Similarities by Multiple Sequence Aalignment," Bioinformatics, vol. 14, no. 3, pp. 290-294, 1998.

[32] J. Thompson, P. Koehl, R. Ripp, and O. Poch, "BAliBASE 3.0: Latest Developments of the Multiple Sequence Alignment Benchmark," Proteins: Structure, Function, and Bioinformatics, vol. 61, no. 1, pp. 127-136, 2005.

[33] G. P. S. Raghava, S. Searle, P. Audley, J. Barber, and G. Barton, "OXBench: A Benchmark for Evaluation of Protein Multiple Sequence Alignment Accuracy," BMC bioinformatics, vol. 4, no. 1, p. 47, 2003.

[34] R. Edgar, "MSA Benchmark Collection," Available at http://www.drive5. $\mathrm{com} / \mathrm{bench} /$

[35] W. K. Kroeze, D. J. Sheffler, and B. L. Roth, "G-protein-coupled Receptors at a Glance," J. Cell Sci., vol. 116, pp. 4867-4869, 2003.

[36] J. Sauder, J. Arthur, and R. Dunbrack, "Large-scale Comparison of Protein Sequence Alignment Algorithms with Structure Alignments," Proteins, vol. 40, pp. 6-22, 2000

[37] M. Cline, R. Hughey, and K. Karplus, "Predicting Reliable Regions in Protein Sequence Alignments," Bioinformatics, vol. 18, no. 2, pp. 306314,2002

[38] J. Thomas, N. Ramakrishnan, and C. Bailey-Kellogg, "Graphical Models of Residue Coupling in Protein Families," IEEE/ACM Transactions on Computational Biology and Bioinformatics, vol. 5, no. 2, pp. 183-197, 2008.

[39] R. Ranganathan, "Statistical Coupling Analysis," Available at http: //systems.swmed.edu/rr_lab/sca.html.

[40] D. Marks, L. Colwell, R. Sheridan, T. Hopf, A. Pagnani, R. Zecchina, and C. Sander, "Protein 3D Structure Computed from Evolutionary Sequence Variation," PLoS ONE, vol. 6, no. 12, p. e28766, 2011

[41] S. W. Lockless and R. Ranganathan, "Evolutionarily Conserved Pathways of Energetic Connectivity in Protein Families," Science, vol. 286, no. 5438, pp. 295-299, 1999.

[42] N. Halabi, O. Rivoire, S. Leibler, and R. Ranganathan, "Protein Sectors: Evolutionary Units of Three-Dimensional Structure," Cell, vol. 138, pp. 774-786, 2009.

[43] A. Waterhouse, J. Procter, D. Martin, M. Clamp, and G. Barton, "Jalview Version 2 - A Multiple Sequence Alignment Editor and Aanalysis Workbench," Bioinformatics, vol. 25, no. 9, pp. 1189-1191, 2009.

[44] S. Balakrishnan, H. Kamisetty, J. Carbonell, S. Lee, and C. Langmead, "Learning Generative Models for Protein Fold Families," Proteins. Structure, Function, and Bioinformatics, vol. 79, no. 4, pp. 1061-1078, 2011.

[45] J. Thomas, N. Ramakrishnan, and C. Bailey-Kellogg, "Protein Design by Sampling an Undirected Graphical Model of Residue Constraints," IEEE/ACM Transactions on Computational Biology and Bioinformatics, vol. 6 , no. 3, pp. 506-516, 2009.
[46] T. Hopf, L. Colwell, R. Sheridan, B. Rost, C. Sander, and D. Marks, "Three-Dimensional Structures of Membrane Proteins from Genomic Sequencing," Cell, vol. 149, no. 7, pp. 1607-1621, 2012.

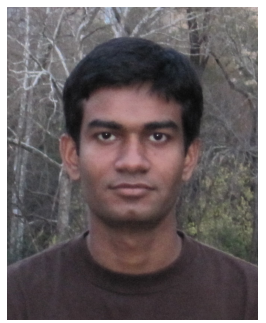

K. S. M. Tozammel Hossain is pursuing Ph.D. degree in computer science at Virginia Tech. He holds an B.S. in computer science \& engineering from Bangladesh University of Engineering \& Technology. His current research interests are in bioinformatics, in particular identifying and modeling evolutionary constraints to assist in protein analysis, classification, and design.

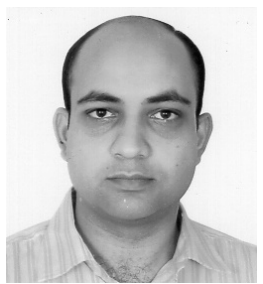

Debprakash Patnaik is currently an engineer in the Search and Discovery group at Amazon.com. He completed his Ph.D. in Computer Science from Virginia Tech, USA. He received his bachelor degree from Veer Surendra Sai University of Technology, Orissa, India, in 2002 and masters degree from Indian Institute of Science, Bangalore, in 2006. $\mathrm{He}$ also worked as a researcher in the Diagnosis and Prognosis group at General Motors Research, Bangalore. His research interests include temporal data mining, probabilistic graphical models, and application in neuroscience.

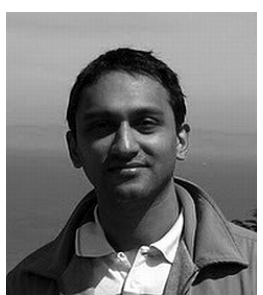

Srivatsan Laxman is a researcher at Microsoft Research India, Bangalore and is associated with two research areas, Machine Learning \& Optimization and Security \& Privacy. He works broadly in data mining, machine learning and data privacy. Srivatsan received his $\mathrm{PhD}$ in Electrical Engineering in 2006 from Indian Institute of Science, Bangalore.

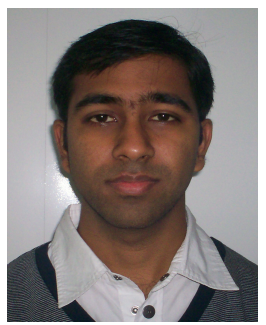

Prateek Jain is a researcher in the Machine Learning and Optimization group at Microsoft Research, Bangalore, India. His research interests lie in the areas of machine learning, optimization, and highdimensional statistics. Before joining Microsoft, Prateek obtained his Ph.D. from the CS department at the University of Texas at Austin. He and his collaborators were recognized with the ICML Best Student Paper Award in 2007, SDM Best Paper Runner-up Award in 2008, and with the CVPR Best Student Paper Award in 2008

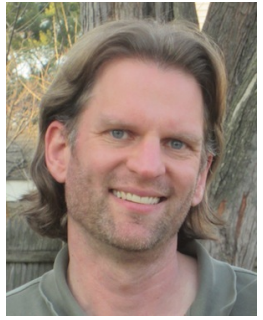

Chris Bailey-Kellogg is an associate professor of computer science at Dartmouth. He earned a BS/MS with Sandy Pentland at MIT and a PhD with Feng Zhao at Ohio State and Xerox PARC, and conducted postdoctoral research with Bruce Donald at Dartmouth. He was an assistant professor at Purdue before being recruited back to Dartmouth. His research focuses on integrating computation and experiment in studies of protein structure and function, with a particular emphasis on applications to protein and vaccine design.

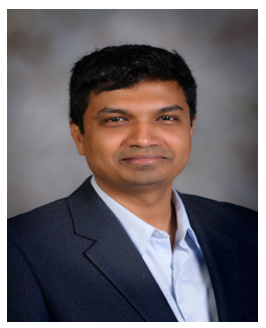

Naren Ramakrishnan is the Thomas L. Phillips Professor of Engineering at Virginia Tech and director of the university's Discovery Analytics Center (DAC; http://dac.cs.vt.edu). His research interests involve mining scientific datasets, in domains such as intelligence analysis, sustainability, neuroscience, and systems biology. Ramakrishnan's work has been supported by NSF, DHS, NIH, NEH, DARPA, IARPA, ONR, General Motors, HP Labs, NEC Labs, and Advance Auto Parts. He received his $\mathrm{PhD}$ in Computer Sciences from Purdue University. 